Article

\title{
Data-Driven Estimation of Significant Kinetic Parameters Applied to the Synthesis of Polyolefins
}

\author{
Santiago D. Salas ${ }^{1,2}$, Amanda L. T. Brandão ${ }^{3}{ }^{\mathbb{C}}$, João B. P. Soares ${ }^{4}$ and José A. Romagnoli ${ }^{1, *}$ \\ 1 Department of Chemical Engineering, Louisiana State University, Baton Rouge, LA 70809, USA; \\ ssalas3@1su.edu \\ 2 Escuela Superior Politécnica del Litoral, ESPOL, Facultad de Ciencias Naturales y Matemáticas, \\ Campus Gustavo Galindo Km. 30.5 Vía Perimetral, P.O. Box 09-01-5863, Guayaquil, Ecuador \\ 3 Engenharia Química, Pontifícia Universidade Católica do Rio de Janeiro, Rio de Janeiro 22451-900, Brazil; \\ amanda.lemette@puc-rio.br \\ 4 Department of Chemical and Materials Engineering, University of Alberta, Edmonton, AB T6G 1H9, Canada; \\ jsoares@ualberta.ca \\ * $\quad$ Correspondence: jose@lsu.edu; Tel.: +1-225-578-1377
}

Received: 23 April 2019; Accepted: 16 May 2019; Published: 22 May 2019

\begin{abstract}
A data-driven strategy for the online estimation of important kinetic parameters was assessed for the copolymerization of ethylene with 1,9-decadiene using a metallocene catalyst at different diene concentrations and reaction temperatures. An initial global sensitivity analysis selected the significant kinetic parameters of the system. The retrospective cost model refinement (RCMR) algorithm was adapted and implemented to estimate the significant kinetic parameters of the model in real time. After verifying stability and robustness, experimental data validated the algorithm performance. Results demonstrate the estimated kinetic parameters converge close to theoretical values without requiring prior knowledge of the polymerization model and the original kinetic values.
\end{abstract}

Keywords: data-driven parameter estimation; retrospective cost model refinement algorithm; global sensitivity analysis; polyolefin synthesis

\section{Introduction}

Polyolefins, mainly polypropylene and polyethylene, are the most common plastics worldwide. The annual growth rate projected for such materials is estimated to be around $3-5 \%$ in the next decade [1], which makes polyolefins a continuously growing and attractive product. Metallocene catalysts such as Dow Chemical's constrained-geometry catalyst (CGC), produce polyolefins with narrow molecular weight distributions (MWD), while allowing the easy addition of $\alpha$-olefins, dienes, and macromonomers into the growing chains [2]. The incorporation of macromonomers generates copolymers with long-chain branches (LCB), which, besides enhancing physical and mechanical properties, improves the processability of the final plastic materials [3-5]. The reaction pathways that lead to the formation of LCBs in ethylene/ $\alpha$-olefins/diene copolymers are complex. Various experimental investigations have studied their polymerization kinetics [6-8], while others have focused on the development of mechanistic models to explain their microstructures and to predict properties of interest [9-12].

Brandão et al. (2017) [13] proposed a mechanistic model for the semi-batch copolymerization of ethylene and 1,9-decadiene with a metallocene catalyst, which was validated using experimental measurements including the ethylene flow rate $\left(F_{M}\right)$, the number-average molecular weight $\left(M_{n}\right)$, and the weight-average molecular weight $\left(M_{w}\right)$. The model assumed that LCBs were formed by incorporating macromonomers through pendant unsaturations resulting from the copolymerization of 
1,9-decadiene. In addition, two methodologies to calculate the polyolefin MWD including the adaptive orthogonal collocation method and Monte-Carlo simulation, were compared [14]. Both methodologies could describe the MWD of polymers made under different experimental conditions, such as reaction temperature and catalyst concentration. For verification, the computed distributions were contrasted with experimental measurements obtained from a high-temperature gel permeation chromatography (GPC) [15].

Even though fundamental models and experimental measurements provide important information to understand the dynamic evolution of a system, neither of them is exact. In real operations, experimental uncertainties may affect previous validations and prevent the model to predict relevant properties accurately. Nonlinear state or parameter estimation strategies, on the other hand, are elegant ways to combine both the experimental data and complex mathematical models [16], which may improve the description of the polymerization system under study. Online estimation techniques are powerful computational tools that can generate immediate knowledge of a particular system of interest, enhance the control actions, and monitor continuously relevant properties [17].

Recent applications of nonlinear estimation methods in polymerization have focused on the reconstruction of the state vector [17-22] rather than on the estimation of polymerization kinetic parameters. Some of the main aims when using state estimators include noise reduction for control purposes, and the prediction of relevant polymer properties such as the MWD, $M_{n}$ and $M_{w}$. Typically, the kinetic parameters of a polymerization model must be estimated before the model can be utilized. The maximum likelihood or the least squares approach are common criteria that aim to match a semi-empirical or fundamental model with the available experimental data. In this context, common strategies include the use of computational packages such as gEST, available in the platform gPROMS [23,24], or metaheuristic/machine-learning algorithms, such as particle swarm optimization or the differential evolution algorithm $[13,25,26]$. These methods, however, achieve a general description of the model, without considering process disturbances, impurities, experimental errors, and side reactions that may occur during the polymerization.

Few authors have studied algorithms for the online estimation of kinetic parameters in polymerization processes. An initial study in this topic was introduced by Sirohi and Choi (1996) [27]. They implemented an extended Kalman (EKF) filter to estimate kinetic parameters and heat transfer coefficients using a computational experiment. Li et al. (2004) [28] employed an EKF for the simultaneous estimation of states and parameters in a continuous reactor for the ethylene-propylene-diene polymerization. Chen et al. (2005) [29] investigated a particle filter strategy in batch polymerization for joint state and parameter estimation. Finally, Sheibat-Othman et al. (2008) [30] compared different online parameter estimation strategies, including the minimization-based approach, EKF, high gain, and adaptive observer. The results were evaluated qualitatively for the solution homopolymerization of acrylic acid using measurements from infrared spectroscopy. However, all of these methods required an adjoint model, or relied on the explicit knowledge of the parameter dependences, which implied that the mathematical model had to be computable and known by the estimation algorithm (e.g., the EKF requires the Jacobian of the states dynamics). In addition, initial information on the parameters was required; otherwise, the algorithm failed to converge [31].

In this contribution, a data-driven online parameter estimation strategy was assessed for the copolymerization of ethylene with 1,9-decadiene using a metallocene catalyst in a semi-batch reactor. The initial phase corresponded to the selection of the significant kinetic parameters of the polymerization model. A global sensitivity analysis that used an improved version of the Sobol method permitted the identification of the most important kinetic parameters of the system [32]. Subsequently, the retrospective cost model refinement (RCMR) algorithm [33-35], a data-driven method that does not require knowledge of the nonlinear model and the initial values of the estimated parameters, was adapted and implemented for the online estimation of the significant kinetic parameters. Different channels allowed the reconstruction of each parameter of interest towards its real value. Finally, the RCMR algorithm was tested and validated with experimental data to verify its applicability. 


\section{Process Modelling}

Fundamental models in polymerization are advantageous tools that contribute to product development and process troubleshooting. The mathematical model described in this work was proposed by Brandão et al. (2017) $[13,14]$. The reaction mechanism adopted for the copolymerization of ethylene with 1,9-decadiene used the catalyst dimethylsilyl ( $N$-tert-butylamido) (tetramethylcyclopentadienyl) titanium dichloride (CGC)/MAO. The reaction mechanism is described as follows:

Catalyst activation

$$
C \stackrel{k_{a}}{\rightarrow} C^{*}
$$

Initiation

$$
C^{*}+M \stackrel{k_{p_{11}}}{\rightarrow} P_{1}^{*}(+m)
$$

Propagation (ethylene)

$$
P_{q}^{*}+M \stackrel{k_{p_{11}}}{\rightarrow} P_{q+1}^{*}(+m)
$$

Propagation (diene)

$$
P_{q}^{*}+D \stackrel{k_{p_{12}}}{\rightarrow} P_{q+1}^{*}(+d)
$$

Transfer to monomer and $\beta$-hydride elimination

$$
P_{q}^{*} \stackrel{k_{t}}{\rightarrow} L_{q}^{=}+C^{*}
$$

Living chain deactivation

$$
P_{q}^{*}+P_{r}^{*} \stackrel{k_{d P}}{\rightarrow} L_{q}+L_{r}+2 D C
$$

Macromonomer reincorporation

$$
P_{q}^{*}+L_{r}^{=} \stackrel{k_{b} K}{\rightarrow} P_{q+r}^{*}(+2 l c b)
$$

where $C$ is the catalyst precursor, $C^{*}$ is an active catalyst site, $M$ is the ethylene, $D$ is the diene, $D C$ is a dead catalyst site, $m$ and $d$ are the total number of ethylene and diene units inserted into the growing polymer chains, $P_{q}^{*}$ is a living polymer chain of size $q, L_{q}^{=}$is a dead polymer chain of size $q$ containing a terminal unsaturation, and $L_{q}$ is a dead polymer chain of size $q$ without a terminal unsaturation.

Under assumptions such as constant ethylene concentration, excess co-catalyst concentration, well-mixed reactor, initiation rate equal to propagation rate for ethylene $\left(k_{p_{11}}\right)$, and propagation controlled by the chemical nature of the monomer species, the set of differential equations that describe the system are shown in Equations (8)-(21).

$$
\begin{gathered}
\frac{\mathrm{d} C}{\mathrm{~d} t}=-k_{a}\left(\frac{C}{V}\right) V \\
\frac{\mathrm{d} C^{*}}{\mathrm{~d} t}=k_{a}\left(\frac{C}{V}\right) V-k_{p_{11}}\left(\frac{C^{*}}{V}\right)\left(\frac{M}{V}\right) V+k_{t}\left(\frac{\mu_{0}}{V}\right) V \\
\frac{\mathrm{d} D C}{\mathrm{~d} t}=k_{d P}\left(\frac{\mu_{0}}{V}\right)^{2} V \\
\frac{\mathrm{d} D}{\mathrm{~d} t}=-k_{p_{12}}\left(\frac{\mu_{0}}{V}\right)\left(\frac{D}{V}\right) V
\end{gathered}
$$




$$
\begin{aligned}
& \frac{\mathrm{d}(m)}{\mathrm{d} t}=k_{p_{11}}\left[\left(\frac{C^{*}}{V}\right)+\left(\frac{\mu_{0}}{V}\right)\right]\left(\frac{M}{V}\right) V \\
& \frac{\mathrm{d}(d)}{\mathrm{d} t}=k_{p_{12}}\left(\frac{\mu_{0}}{V}\right)\left(\frac{D}{V}\right) V \\
& \frac{\mathrm{d}(l c b)}{\mathrm{d} t}=2 k_{b} \varphi\left(\frac{\mu_{0}}{V}\right)\left(\frac{\lambda_{1}}{V}\right) V \\
& \frac{\mathrm{d} \mu_{0}}{\mathrm{~d} t}=-k_{t}\left(\frac{\mu_{0}}{V}\right) V-k_{d P}\left(\frac{\mu_{0}}{V}\right)^{2} V+k_{p_{11}}\left(\frac{C^{*}}{V}\right)\left(\frac{M}{V}\right) V \\
& \frac{\mathrm{d} \mu_{1}}{\mathrm{~d} t}=-k_{t}\left(\frac{\mu_{1}}{V}\right) V-k_{d P}\left(\frac{\mu_{0}}{V}\right)\left(\frac{\mu_{1}}{V}\right) V+k_{p_{11}}\left[\left(\frac{C^{*}}{V}\right)+\left(\frac{\mu_{0}}{V}\right)\right]\left(\frac{M}{V}\right) V+k_{p_{12}}\left(\frac{\mu_{0}}{V}\right)\left(\frac{D}{V}\right) V \\
& +k_{b} \varphi\left(\frac{\mu_{0}}{V}\right)\left(\frac{\lambda_{2}}{V}\right) V \\
& \frac{\mathrm{d} \mu_{2}}{\mathrm{~d} t}=-k_{t}\left(\frac{\mu_{2}}{V}\right) V-k_{d P}\left(\frac{\mu_{0}}{V}\right)\left(\frac{\mu_{2}}{V}\right) V+k_{p_{11}}\left[\left(\frac{\mathrm{C}^{*}}{V}\right)+\left(\frac{\mu_{0}}{V}\right)+2\left(\frac{\mu_{1}}{V}\right)\right]\left(\frac{M}{V}\right) V \\
& +k_{p_{12}}\left[\left(\frac{\mu_{0}}{V}\right)+2\left(\frac{\mu_{1}}{V}\right)\right]\left(\frac{D}{V}\right) V+k_{b} \varphi\left[\left(\frac{\mu_{0}}{V}\right)\left(\frac{\lambda_{3}}{V}\right)+2\left(\frac{\mu_{1}}{V}\right)\left(\frac{\lambda_{2}}{V}\right)\right] V \\
& \frac{\mathrm{d} \lambda_{0}}{\mathrm{~d} t}=k_{t}\left(\frac{\mu_{0}}{V}\right) V+k_{d P}\left(\frac{\mu_{0}}{V}\right)^{2} V-k_{b} \varphi\left(\frac{\mu_{0}}{V}\right)\left(\frac{\lambda_{1}}{V}\right) V \\
& \frac{\mathrm{d} \lambda_{1}}{\mathrm{~d} t}=k_{t}\left(\frac{\mu_{1}}{V}\right) V+k_{d P}\left(\frac{\mu_{0}}{V}\right)\left(\frac{\mu_{1}}{V}\right) V-k_{b} \varphi\left(\frac{\mu_{0}}{V}\right)\left(\frac{\lambda_{2}}{V}\right) V \\
& \frac{\mathrm{d} \lambda_{2}}{\mathrm{~d} t}=k_{t}\left(\frac{\mu_{2}}{V}\right) V+k_{d P}\left(\frac{\mu_{0}}{V}\right)\left(\frac{\mu_{2}}{V}\right) V-k_{b} \varphi\left(\frac{\mu_{0}}{V}\right)\left(\frac{\lambda_{3}}{V}\right) V \\
& \frac{\mathrm{d} V}{\mathrm{~d} t}=\left(\frac{d \lambda_{1}}{d t}+\frac{d \mu_{1}}{d t}\right) \frac{M M}{\rho_{P E}}
\end{aligned}
$$

where,

$$
\begin{gathered}
\varphi=\frac{d}{d+m} \\
\lambda_{3}=\frac{\lambda_{2}}{\lambda_{0} \lambda_{1}}\left(2 \lambda_{2} \lambda_{0}-\lambda_{1}^{2}\right) \\
M M=\varphi M M_{D}+(1-\varphi) M M_{M}
\end{gathered}
$$

When the polymerization mechanism leads to moment closure problems, the $q^{\text {th }}$-moment balance equation requires the definition of the $(q+1)^{t h}$ moments. Otherwise, the balance equation cannot be solved. Hulburt and Katz (1964) [36] developed a closure method that can be written in the form of algebraic expressions, using a distribution approximation procedure. The closure expression for $\lambda_{3}$ was then obtained as approximate algebraic equation in the form of Equation (23).

The concentration of ethylene was kept constant during the polymerization, $\frac{\mathrm{d} M}{\mathrm{~d} t}=0$. Thus, the inlet flow rate of ethylene $\left(F_{M}\right)$, which represents the continuous demand of ethylene during the polymerization, was approximated to the expected demand of monomer during the reaction, as denoted in Equation (25).

$$
F_{M} \approx k_{p_{11}}\left(\frac{C^{*}}{V}\right)\left(\frac{M}{V}\right) V+k_{p_{11}}\left(\frac{\mu_{0}}{V}\right)\left(\frac{M}{V}\right) V
$$

The average properties of the resultant polymers were computed as written in Equations (26)-(28).

$$
M_{n}=\frac{\lambda_{1}+\mu_{1}}{\lambda_{0}+\mu_{0}} M M
$$




$$
\begin{gathered}
M_{w}=\frac{\lambda_{2}+\mu_{2}}{\lambda_{1}+\mu_{1}} M M \\
P D I=\frac{M_{w}}{M_{n}}=\frac{\lambda_{2}+\mu_{2}}{\lambda_{0}+\mu_{0}}
\end{gathered}
$$

The fundamental model relied on both the reparametrized and the classical Arrhenius law to compute the rate constants as listed in Table 1.

Table 1. Kinetic rate constants for the copolymerization of ethylene and 1,9-decadiene.

\begin{tabular}{cl}
\hline Rate Constant & \multicolumn{1}{c}{ Arrhenius Equation } \\
\hline Catalyst activation & $k_{a}=\exp \left[k_{1}+k_{2}\left(\frac{T-T_{r}}{T}\right)\right]$ \\
Propagation & $k_{p_{11}}=k_{0 p} \exp \left(-\frac{E_{a p}}{R T}\right), k_{0 p}=10^{k_{7}}$ \\
Monomer transfer \& $\beta$-hydride elimination & $k_{t}=\exp \left[k_{3}+k_{4}\left(\frac{T-T_{r}}{T}\right)\right]$ \\
Living chain deactivation & $k_{d P}=\exp \left[k_{5}+k_{6}\left(\frac{T-T_{r}}{T}\right)\right]$ \\
\hline
\end{tabular}

$T$ : Temperature inside the reactor, $T_{r}$ : reference temperature set to $130^{\circ} \mathrm{C}$.

The molar concentration and total amount of monomer, listed in Table 2, were obtained using the Peng-Robinson equation to calculate fugacity, and UNIQUAC model to determine the activity coefficients in the liquid phase.

Table 2. Ethylene concentration and total moles of ethylene in toluene at different temperatures.

\begin{tabular}{ccc}
\hline $\mathbf{T},\left[\mathbf{C}^{\circ}\right]$ & {$\left[\mathbf{C}_{\mathbf{2}} \mathbf{H}_{\mathbf{4}}\right],\left[\mathbf{m o l ~ L}^{-\mathbf{1}}\right]$} & {$[\mathbf{M}],[\mathbf{m o l}]$} \\
\hline 120 & 0.49472 & 0.07420 \\
130 & 0.43732 & 0.06560 \\
140 & 0.38141 & 0.05721 \\
\hline
\end{tabular}

The parameters $k_{1-7}$ had their values determined stochastically by using particle swarm optimization (PSO) [37] in the homopolymerization experiments. After this procedure, the identifiability analysis indicated that only four parameters $\left(k_{3}, k_{5}, k_{6}\right.$ and $\left.k_{7}\right)$ could be estimated simultaneously. The remaining parameters $\left(k_{1}, k_{2}\right.$ and $\left.k_{4}\right)$, although important for model computations, could not be estimated independently with the available data; therefore, their values were kept constant and equal to the values provided by the PSO. Then, the four selected parameters were estimated using the computational package ESTIMA [25]. The experimental data used to estimate the parameters included the average properties $M_{n}$ and $M_{w}$, and the ethylene feed rates $\left(F_{M}\right)$, which was the only continuous measurement. The copolymerization kinetic parameters $k_{p_{12}}$ and $k_{b}$ were estimated by ESTIMA considering the experimental data of $M_{n}$ and $M_{w}$ only.

Table 3 lists the parameters of the system, including the kinetic parameters in the reparametrized Arrhenius equations, pre-exponential constants, activation energies, as well as other relevant thermodynamic properties and constants. It is important to remind the reader that the kinetic parameters $\left(k_{1-7}\right)$ are needed to describe the actual rate constants. Table 4 provides the initial conditions considered in the current investigation. The interested reader is encouraged to consult the original publication for more details [13]. A complete explanation of the variables and kinetic parameters is included in the Nomenclature. 
Table 3. Parameters of the copolymerization of ethylene with 1,9-decadiene using dimethylsilyl (N-tert-butylamido) (tetramethylcyclopentadienyl) titanium dichloride (CGC)/MAO.

\begin{tabular}{cccc}
\hline Parameter & Value & Units & Original Rate Constants $^{\mathbf{1}}$ \\
\hline$k_{1}$ & -2.92 & - & $k_{a}=\alpha\left(k_{1}, k_{2}\right)$ \\
$k_{2}$ & 25.00 & - & $k_{t}=\alpha\left(k_{3}, k_{4}\right)$ \\
$k_{3}$ & $2.58 \pm 0.08$ & - & $k_{d P}=\alpha\left(k_{5}, k_{6}\right)$ \\
$k_{4}$ & 17.2 & - & $k_{p_{11}}=\alpha\left(k_{7}\right)$ \\
$k_{5}$ & $10.91 \pm 0.95$ & - & - \\
$k_{6}$ & $30.12 \pm 2.10$ & - & - \\
$k_{7}$ & $7.56 \pm 0.07$ & - & - \\
$k_{p_{12}}$ & $2039.8 \pm 54.7$ & $\mathrm{~L} \mathrm{~mol}^{-1} \mathrm{~s}^{-1}$ & - \\
$k_{b}$ & $908.7 \pm 69.0$ & $\mathrm{~L} \mathrm{~mol}^{-1} \mathrm{~s}^{-1}$ & - \\
$E_{a p}$ & 20520.0 & $\mathrm{~J} \mathrm{~mol}^{-1}$ & - \\
$M M_{M}$ & 28.05 & $\mathrm{~g} \mathrm{~mol}^{-1}$ & - \\
$M M_{D}$ & 138.254 & $\mathrm{~g} \mathrm{~mol}^{-1}$ & $\mathrm{~g} \mathrm{~L}^{-1}$ \\
$\rho_{P E}$ & 940 & $\mathrm{~J} \mathrm{~mol}^{-1} \mathrm{~K}^{-1}$ & \\
$R$ & 8.31451 & ${ }_{k}=\alpha\left(k_{\beta}, k_{\gamma}\right)$ represents $k_{\iota}$ as a function of $k_{\beta}$ and $k_{\gamma}$.
\end{tabular}

Table 4. Initial conditions for simulations and experiments.

\begin{tabular}{ccccc}
\hline \multirow{2}{*}{ Variable } & Homopolymerization & \multicolumn{2}{c}{ Copolymerization } & Units \\
\cline { 3 - 4 } & & A & B & \\
\hline$C(0)$ & $0.767 \times 10^{-6} \times \mathrm{V}(0)$ & $0.271 \times 10^{-6} \times \mathrm{V}(0)$ & $0.271 \times 10^{-6} \times \mathrm{V}(0)$ & $\mathrm{mol}$ \\
$C^{*}(0)$ & 0 & 0 & 0 & $\mathrm{~mol}$ \\
$D C(0)$ & 0 & 0 & 0 & $\mathrm{~mol}$ \\
$D(0)$ & 0 & $0.3 \div M M_{D}$ & $0.4 \div M M_{D}$ & $\mathrm{~mol}$ \\
$(M)(0)$ & 0 & 0 & 0 & $\mathrm{~mol}$ \\
$(d)(0)$ & 0 & 0 & 0 & $\mathrm{~mol}$ \\
$(l c b)(0)$ & 0 & 0 & 0 & $\mathrm{~mol}$ \\
$\mu_{0}(0)$ & 0 & 0 & 0 & $\mathrm{~mol}$ \\
$\mu_{1}(0)$ & 0 & 0 & 0 & $\mathrm{~mol}$ \\
$\mu_{2}(0)$ & 0 & 0 & 0 & $\mathrm{~mol}$ \\
$\lambda_{0}(0)$ & $10^{-14}$ & $10^{-14}$ & $10^{-14}$ & $\mathrm{~mol}$ \\
$\lambda_{1}(0)$ & $10^{-14}$ & $10^{-14}$ & $10^{-14}$ & $\mathrm{~mol}$ \\
$\lambda_{2}(0)$ & $10^{-14}$ & $10^{-14}$ & 0.15 & $\mathrm{~mol}$ \\
$V(0)$ & 0.15 & 0.15 & & $\mathrm{~L}$ \\
\hline
\end{tabular}

\section{Experimental Equipment and Setup}

\subsection{Materials}

The materials utilized in the experimental runs were methylaluminoxane (MAO, $10 \mathrm{wt}$. \% in toluene), anhydrous ethyl alcohol ( $\geq 99.5 \%)$, toluene anhydrous (99.8\%), 1,9-Decadiene (98\%), triisobutylaluminum (TIBA) (25 wt. \% in toluene), n-butyllithium solution (2.5 M in hexane), sodium ( $\geq 99 \%$, stored in mineral oil), which were provided by Sigma-Aldrich (USA). Moreover, dimethylsily (n-tert-butylamido) (tetramethylcyclopentadienyl) titanium dichloride (CGC) (85.0-99.8\%) was acquired from Boulder Scientific Company (USA), and nitrogen (>99.998\%) and ethylene were provided by Praxair Technology (USA).

\subsection{Process Description}

Prior polymerization, six cycles of nitrogen venting and vacuuming at $125^{\circ} \mathrm{C}$ were applied in the reactor to remove oxygen. Then, the reactor received $150 \mathrm{~mL}$ of toluene and $0.5 \mathrm{~g}$ of TIBA (impurity scavenger), and the temperature was increased to $120^{\circ} \mathrm{C}$ and kept constant for $20 \mathrm{~min}$.

For homopolymerization, after the reactor purging, $150 \mathrm{~mL}$ of toluene was charged at ambient temperature. A solution of MAO was added into the reactor through a cannula under nitrogen 
pressure. The reactor was then heated until reaching the reaction temperature $\left(120,130\right.$ or $\left.140{ }^{\circ} \mathrm{C}\right)$. Then, ethylene was injected into the reactor until the solvent was saturated. After stabilizing the temperature, the catalyst solution was added into the reactor under nitrogen pressure. During polymerization, the reactor temperature remained constant, with variations of $\pm 0.15^{\circ} \mathrm{C}$ from the set point. Ethylene was supplied on demand, maintaining a constant reactor pressure (120 psig). When the final reaction time was achieved, the ethylene supply valve was closed, and the reactor contents were immediately transported into a $1 \mathrm{~L}$ beaker with $100-250 \mathrm{~mL}$ of ethanol. Afterwards, the polymer was kept overnight under constant stirring, then filtered and dried in an oven. The copolymerization procedure was analogous to the homopolymerization procedure. The unique difference is that after adding MAO, the co-monomer solution was injected into the reactor following the same procedure used to feed MAO.

The average properties and the molecular weight distributions of the polymer samples were measured using a Polymer Char High-Temperature Gel Permeation Chromatographer (GPC) calibrated with polystyrene narrow standards and using a universal calibration curve in accordance with the methodology described by Soares and McKenna (2013) [15].

\section{Data-Driven Estimation of Significant Kinetic Parameters}

\subsection{Parameter Selection: Global Sensitivity Analysis}

A global sensitivity analysis shows how significant inputs are with respect to one or various outputs. A robust and widely used variance-based sensitivity analysis is the Sobol method [38]. This method proposes the expansion of a function $G=g\left(z_{1}, \ldots, z_{j}, \ldots, z_{J}\right)$ into terms of increasing dimensions with mutually independent input parameters such that all summands are mutually orthogonal, as explained in Equation (29).

$$
G=g_{0}+\sum_{j=1}^{J} g_{j}\left(z_{j}\right)+\sum_{1 \leq j<b \leq \mathrm{J}} g_{j b}\left(z_{j}, z_{b}\right)+\cdots+g_{1,2, \cdots, J}\left(z_{1}, \ldots, z_{J}\right)
$$

where the index $j$ denotes a parameter of interest, $b$ another parameter, and $J$ is the total number of evaluated parameters. Each term in Equation (29) has quadratic integrability over the domain of existence, where $g_{0}$ is a constant, $g_{j}=g_{j}\left(z_{j}\right), g_{j b}=g_{j b}\left(z_{j}, z_{b}\right)$, and so forth. Equation (30) shows the decomposition of the variance of $G$.

$$
V(G)=\sum_{\mathrm{j}=1}^{J} V_{j}+\sum_{1 \leq \mathrm{j}<b \leq \mathrm{J}} V_{j b}+\ldots+V_{1, \ldots, \mathrm{j}, \ldots, \mathrm{J}}
$$

where $V_{j}, V_{j b}, V_{1, \cdots, j, \cdots, \mathrm{J}}$ are the individual variances of functions $g_{j}, g_{j b}, g_{1}, \cdots, \mathrm{j}, \cdots, \mathrm{J}$.

Sensitivity indices help understand the variance decomposition from Equation (30). First-order sensitivity indices $\left(\hat{S}_{j}\right)$ permit the selection and classification of the most sensitive parameters, depending on the individual importance of their contribution in changing the variance of the function of interest. The main effect of varying parameter $z_{j}$ on the output value $G$ is measured by $\hat{S}_{j}$, as presented in Equation (31). In addition, the total sensitivity index $\left(\hat{S}_{T j}\right)$ incorporates the sum of all the effects that involve the parameter $z_{j}$. The total sensitivity index for parameter $z_{j}$ is computed as indicated in Equation (32).

$$
\begin{gathered}
\hat{S}_{j}=\frac{\hat{V}_{j}}{\hat{V}} \\
\hat{S}_{T j}=1-\frac{\hat{V}_{-j}}{\hat{V}}
\end{gathered}
$$

where $\hat{V}_{-j}$ is the sum of all variance terms that exclude $z_{j}$. 
$\hat{S}_{j}$ and $\hat{S}_{T j}$ can be compared to evaluate whether a model is additive or not. For non-additive models, $\hat{S}_{j}<\hat{S}_{T j}$; for additive models, $\hat{S}_{j}=\hat{S}_{T j}$. Additive models are those in which no interactions between evaluated parameters occur [39].

The Sobol standard method may be improved by introducing sampling and resampling matrices [40,41], and even better performance is achieved when the results of the evaluated functions are averaged, creating extra data points [32]. In this study, we used an improved version of Sobol's method, as implemented by Salas et al. (2017) [42], including a third sampling matrix to avoid unfeasible scenarios. The method follows the steps below:

(1) Define an objective function, and the dimension $(D)$ for a sample of input parameters. For each parameter, define an uncertainty index. In this case, we adopted $4 \%$ of change with respect to the mean value.

(2) Build three random matrices $\boldsymbol{M}_{\mathbf{1}}, \boldsymbol{M}_{\mathbf{2}}$ and $\boldsymbol{M}_{3}$-Equations (33a)-(33c), respectively-of dimension $D \times J$ based on the defined uncertainty: $\boldsymbol{M}_{\mathbf{1}}$ is the sampling matrix, $\boldsymbol{M}_{\mathbf{2}}$ is the resampling matrix, and $M_{3}$ is the backup matrix.

$$
\begin{aligned}
& \boldsymbol{M}_{\mathbf{1}}=\left[\begin{array}{ccc}
z_{11} \cdots & z_{1 j} \cdots & z_{1 J} \\
\vdots & \vdots & \vdots \\
z_{D 1} \cdots & z_{D j} \cdots & z_{D J}
\end{array}\right] \\
& \boldsymbol{M}_{\mathbf{2}}=\left[\begin{array}{ccc}
z_{11}^{\prime} \cdots & z_{1 j}^{\prime} \cdots & z_{1 J}^{\prime} \\
\vdots & \vdots & \vdots \\
z_{D 1}^{\prime} \cdots & z_{D j}^{\prime} \cdots & z_{D J}^{\prime}
\end{array}\right] \\
& \boldsymbol{M}_{3}=\left[\begin{array}{ccc}
z_{11}^{\prime \prime} \cdots & z_{1 j}^{\prime \prime} \cdots & z_{1 J}^{\prime \prime} \\
\vdots & \vdots & \vdots \\
z_{D 1}^{\prime \prime} \cdots & z_{D j}^{\prime \prime} \cdots & z_{D J}^{\prime \prime}
\end{array}\right]
\end{aligned}
$$

(3) Evaluate the row vectors of matrices $\boldsymbol{M}_{\mathbf{1}}$ and $\boldsymbol{M}_{\mathbf{2}}$. If the output is unfeasible, meaning that the combination of inputs in a vector caused the simulation to break or other related problems, use the next available feasible row of the matrix $M_{3}$, and update the matrices to $M_{1}$ ' and $M_{2}$, which denote the improved sampling and resampling matrices, respectively. Then, calculate the total average $\left(\hat{g}_{0}\right)$ of both evaluations as described in Equation (34).

$$
\begin{gathered}
g_{S}=g\left(\boldsymbol{M}^{\prime}\right), \quad g_{\boldsymbol{R}}=g\left(\boldsymbol{M}_{\mathbf{2}^{\prime}}\right) \\
\hat{g}_{0}=\frac{1}{2 D} \sum_{d=1}^{D}\left(g_{S}+g_{\boldsymbol{R}}\right)
\end{gathered}
$$

where $g_{S}$ represents the output vector of $\boldsymbol{M}_{\mathbf{1}^{\prime}}$ and $g_{R}$ is the output vector of $\mathbf{M}_{\mathbf{2}^{\prime}}$.

(4) Generate a matrix $N_{q}$ formed by all columns of matrix $M_{2^{\prime}}$, except the column of the $z_{\mathrm{q}}$ parameter, which is pulled from $\boldsymbol{M}_{\mathbf{1}^{\prime}}$, as explained in Equation (35a). Subsequently, generate another matrix $\boldsymbol{N}_{T j}$ formed with all columns of $\boldsymbol{M}_{\mathbf{1}^{\prime}}$ and with the column of the $z_{j}^{\prime}$ parameter, pulled from $\boldsymbol{M}_{\mathbf{2}^{\prime}}$ as denoted in Equation (35b).

$$
N_{j}=\left[\begin{array}{ccc}
z_{11}^{\prime} \cdots & z_{1 j} \cdots & z_{1 J}^{\prime} \\
\vdots & \vdots & \vdots \\
z_{D 1}^{\prime} \cdots & z_{D j} \cdots & z_{D J}^{\prime}
\end{array}\right]
$$




$$
N_{T j}=\left[\begin{array}{ccc}
z_{11} \cdots & z_{1 j}^{\prime} \cdots & z_{1 J} \\
\vdots & \vdots & \vdots \\
z_{D 1} \cdots & z_{D j}^{\prime} \cdots & z_{D J}
\end{array}\right]
$$

(5) Evaluate the row vectors of matrices $N_{j}$ and $N_{T j}$. If an evaluated function is unfeasible, the output is replaced by $\hat{g}_{0}$. The outputs are obtained in column vectors.

$$
g_{j}^{\prime}=g\left(N_{j}\right), g_{R j}^{\prime}=g\left(N_{T j}\right)
$$

where $g_{j}^{\prime}$ is the output vector of matrix $N_{j}$, and $g_{R j}^{\prime}$ is the output vector of matrix $N_{T j}$.

(6) A sample generates the following estimates, which are calculated based on scalar products of the vectors from above.

$$
\begin{gathered}
\gamma_{q}^{2}=\frac{1}{2 D} \sum_{d=1}^{D}\left(g_{S} \cdot g_{R}+g^{\prime} \cdot g_{R j}^{\prime}\right) \\
\hat{V}=\frac{1}{2 D} \sum_{d=1}^{D}\left(g_{S}{ }^{2}+g_{R}{ }^{2}\right)-\hat{g}_{0}^{2} \\
\hat{V}_{q}=\frac{1}{2 D} \sum_{d=1}^{D}\left(g_{S} \cdot g_{R j}^{\prime}+g_{R} \cdot g_{j}^{\prime}\right)-\gamma_{j}^{2} \\
\hat{V}_{-q}=\frac{1}{2 D} \sum_{d=1}^{D}\left(g_{S} \cdot g_{j}^{\prime}+g_{R} \cdot g_{R j}^{\prime}\right)-\gamma_{j}^{2}
\end{gathered}
$$

where $\gamma_{j}^{2}$ is the squared mean value of the outputs for each parameter $z_{j}$. The selection of sensitive parameters relies on the first and total sensitivity indices. Equation (40) introduces the objective function, defined in this case as:

$$
G=\sum_{i=1}^{i} \frac{y_{i}-h_{i}(z)}{\sigma_{y_{i}}^{2}}
$$

where $y_{i}$ is the measurement at each time interval $i, h_{i}(z)$ is the calculated measurement, and $\sigma_{y_{i}}^{2}$ is the variance of the experimental fluctuations.

\subsection{Data-Driven Parameter Estimation}

\subsubsection{Estimation Problem}

The polymerization model described in Equations (8)-(21) can be written in compact discrete-time form as portrayed in Equation (41).

$$
\left\{\begin{array}{c}
x_{i+1}=f\left(x_{i}, u_{i}, z\right)+v_{i} \\
y_{i}=h\left(x_{i}, u_{i}, z\right)+w_{i}
\end{array}\right.
$$

where $x \in \mathbb{R}^{l_{x}}$ is the state vector, $u \in \mathbb{R}^{l_{u}}$ is the vector of inputs, $z \in \mathbb{R}^{l_{\mu}}$ is the unknown parameter vector, $y \in \mathbb{R}^{l_{y}}$ is the vector of measurements, and $v \in \mathbb{R}^{l_{x}}$ and $w \in \mathbb{R}^{l_{y}}$ are the model and measurement errors, respectively.

For estimation purposes, the compact model is considered to be as shown in Equation (42).

$$
\left\{\begin{array}{c}
\hat{x}_{i+1}=f\left(\hat{x}_{i}, u_{i}, \hat{z}\right)+\boldsymbol{v}_{i} \\
\hat{y}_{i}=h\left(\hat{x}_{i}, \boldsymbol{u}_{i}, \hat{z}\right)+w_{i}
\end{array}\right.
$$


where $\hat{x}$ is the estimated state vector, $\hat{y}$ is the vector of estimated measurements, and $\hat{z}$ is the output of the parameter estimator. The parameter estimator is updated by minimizing a cost function based on the performance variable $(e)$.

Considering an ARMA model with a built-in integrator, $\hat{z}$ is given by:

$$
\hat{z}_{i}=\sum_{l=1}^{n_{c}} \boldsymbol{P}_{l i} \hat{z}_{i-1}+\sum_{l=1}^{n_{c}} \boldsymbol{Q}_{l i} \boldsymbol{e}_{i-l}+\boldsymbol{R}_{i} \boldsymbol{g}_{i}
$$

where,

$$
\begin{gathered}
\boldsymbol{g}_{i}=\boldsymbol{g}_{i-1}+\boldsymbol{e}_{i-1} \\
\boldsymbol{e}_{i}=\hat{\boldsymbol{y}}_{i}-\boldsymbol{y}_{i}
\end{gathered}
$$

and $\boldsymbol{P}_{i} \in \mathbb{R}^{l_{z} \times l_{z}}, \boldsymbol{Q}_{i}, \boldsymbol{R} \in \mathbb{R}^{l_{z} \times l_{y}}$ are the coefficient matrices that are updated recursively by the algorithm. The integrator is combined with the estimator to guarantee that the performance variable approaches to zero as the iterations approach to infinity. Rewriting Equation (43), the following is obtained:

$$
\hat{z}_{i}=\boldsymbol{\Phi}_{i} \theta_{i}
$$

where,

$$
\begin{gathered}
\boldsymbol{\Phi}_{i}=\mathbf{I}_{l_{z}} \otimes \boldsymbol{\phi}_{i}^{T} \in \mathbb{R}^{l_{z} \times l_{\theta}}, \\
\boldsymbol{\phi}_{i}=\left[\hat{z}_{i-1}{ }^{T} \ldots \hat{z}_{i-n_{c}}{ }^{T} \boldsymbol{e}_{i-1}{ }^{T} \ldots \boldsymbol{e}_{i-n_{c}}{ }^{T} \boldsymbol{g}_{i}^{T}\right]^{T} \\
\boldsymbol{\theta}_{i}=\operatorname{vec}\left[\boldsymbol{P}_{1 i} \ldots \boldsymbol{P}_{n_{c} i} \boldsymbol{Q}_{1 i} \ldots \boldsymbol{Q}_{n_{c}} \boldsymbol{R}_{i}\right] \in \mathbb{R}^{l_{\theta}} \\
l_{\theta}=l_{z}{ }^{2} n_{c}+l_{z} l_{y}\left(n_{c}+1\right)
\end{gathered}
$$

and $\boldsymbol{\Phi}$ is the regressor matrix, $\theta$ contains the estimator coefficients computed by the RCMR algorithm. The operator " $\otimes$ " is the Kronecker product, and vec represents the column-stacking operator.

It is assumed that $z$ is identifiable, which is guaranteed from the global sensitivity analysis (in the absence of an observability/detectability analysis), and that the input signal $\boldsymbol{u}_{\boldsymbol{k}}$ is persistently exciting.

\subsubsection{Retrospective Cost Model Refinement (RCMR) Algorithm}

The retrospective performance variable is defined as follows:

$$
\hat{\boldsymbol{e}}_{i}=\boldsymbol{e}_{i}-\boldsymbol{G}_{f}(q)\left(\boldsymbol{\Phi}_{i} \hat{\theta}-\hat{z}_{i}\right)
$$

where $q$ is the forward-shift operator, and $\hat{\theta}$ has the parameter estimation coefficients to be optimized.

$$
G_{f}(q)=\sum_{n=1}^{n_{f}} \frac{N_{n}}{q^{n}}
$$

For all $n, N_{n} \in \mathbb{R}^{l_{y} \times l_{z}} . G_{f}$ is a finite impulse response filter of order $n_{f}$. Equation (47) is then rewritten as follows:

$$
\hat{\boldsymbol{e}}_{i}=\boldsymbol{e}_{i}+N \Phi_{b i} \hat{\theta}-N Z_{b i}
$$

where,

$$
N=\left[N_{1} \ldots N_{n_{f}}\right] \in \mathbb{R}^{l_{y} \times l_{z} n_{f}},
$$




$$
\begin{gathered}
\boldsymbol{\Phi}_{b i}=\left[\begin{array}{c}
\phi_{i-1} \\
\vdots \\
\phi_{i-n_{f}}
\end{array}\right] \in \mathbb{R}^{l_{z} n_{f} \times l_{\theta}}, \\
\mathbf{Z}_{b i}=\left[\begin{array}{c}
\hat{z}_{i-1} \\
\vdots \\
\hat{z}_{i-n_{f}}
\end{array}\right] \epsilon \mathbb{R}^{l_{z} n_{f}} .
\end{gathered}
$$

The retrospective cost function, defined by Goel and Bernstein (2018) [34,35], is minimized by making use of recursive least squares. Let $\mathbf{P}_{0}=\mathbf{R}_{\theta}{ }^{-1}$ and $\theta_{i}=0$.

The algorithm that updates the estimator coefficients is as follows:

$$
\begin{gathered}
\boldsymbol{\theta}_{i}=\boldsymbol{\theta}_{i-1}-\mathbf{P}_{i} \boldsymbol{\Phi}_{b i-1}{ }^{\mathrm{T}} \boldsymbol{N}^{\mathrm{T}} \mathbf{R}_{\mathrm{e} i-1}\left(\mathrm{~N} \boldsymbol{\Phi}_{b i-1} \theta_{i-1}+\boldsymbol{e}_{i-1}-N \mathbf{Z}_{b i-1}\right) \\
\mathbf{P}_{i}=\lambda^{-1} \mathbf{P}_{i-1}-\lambda^{-1} \mathbf{P}_{i-1} \boldsymbol{\Phi}_{b i-1}{ }^{\mathrm{T}} \boldsymbol{N}^{\mathrm{T}} \Gamma_{i}^{-1} \boldsymbol{N} \boldsymbol{\Phi}_{b i-1} \mathbf{P}_{i-1} \\
\Gamma_{i}=\lambda \mathbf{R}_{\mathrm{e} i-1}{ }^{-1}+\boldsymbol{N} \boldsymbol{\Phi}_{b i-1} \mathbf{P}_{i-1} \boldsymbol{\Phi}_{b i-1}{ }^{\mathrm{T}} \boldsymbol{N}^{\mathrm{T}}
\end{gathered}
$$

where $\mathbf{R}_{\mathrm{e}}$ and $\mathbf{R}_{\theta}$ are positive-definite matrices, and $\lambda \leq 1$ is the forgetting factor.

\subsection{Framework Implementation}

The assembly of the implemented strategies is summarized in Figure 1. Initially, the global sensitivity analysis provides information on the most important parameters of the polymerization system. Once these parameters are identified, the proposed framework tries to estimate their values asynchronously, updating/estimating the parameters whenever measurements are available. Monitoring and signal processing are other challenges of the proposed methodology. The estimated properties are expected to be close to the experimental and theoretical values, and noise reduction of the measurements is anticipated to occur.

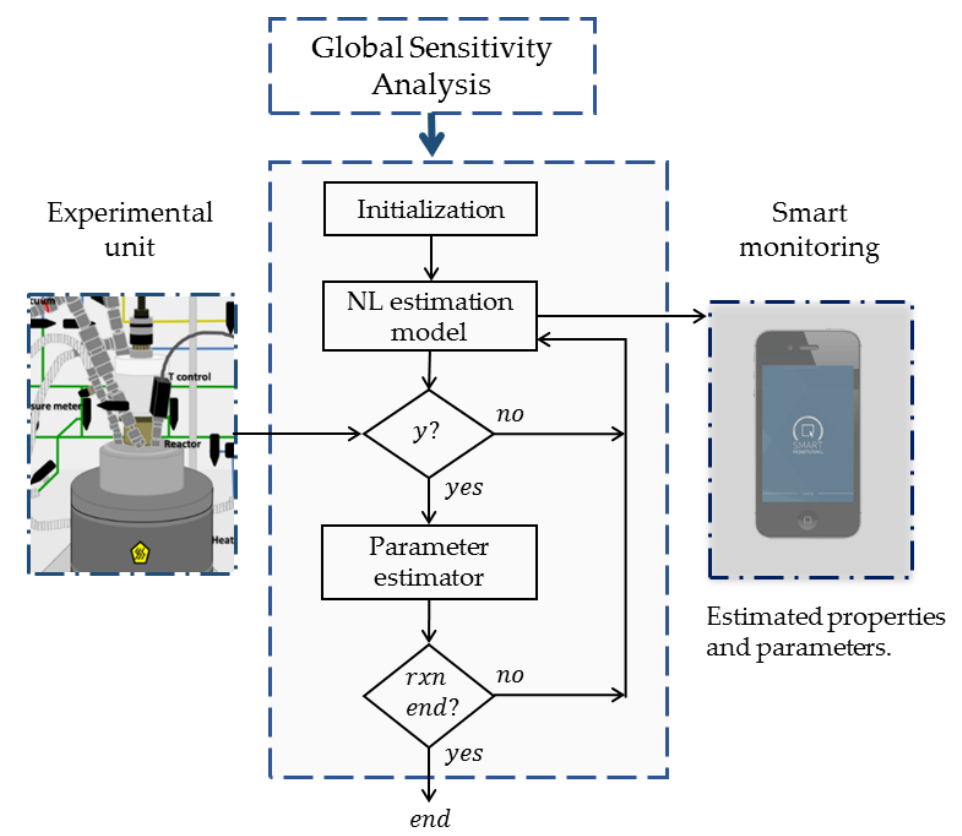

Figure 1. Framework for the online estimation of significant kinetic parameters. 
Figure 2 portrays the architecture of the data-driven estimation strategy. The experimental unit generates measurements $y$, which are assumed to be driven by the inputs $u$. The data-driven adaptive estimator consists of the nonlinear estimation model, which is also driven by the inputs $u$, and the RCMR algorithm. Although the nonlinear estimation model is required to generate the estimated measurements $\hat{y}$, it does not provide knowledge for the parameter updates. The estimated parameter $\hat{z}$ is updated by the estimator, which seeks the minimization of the error signal $e$.

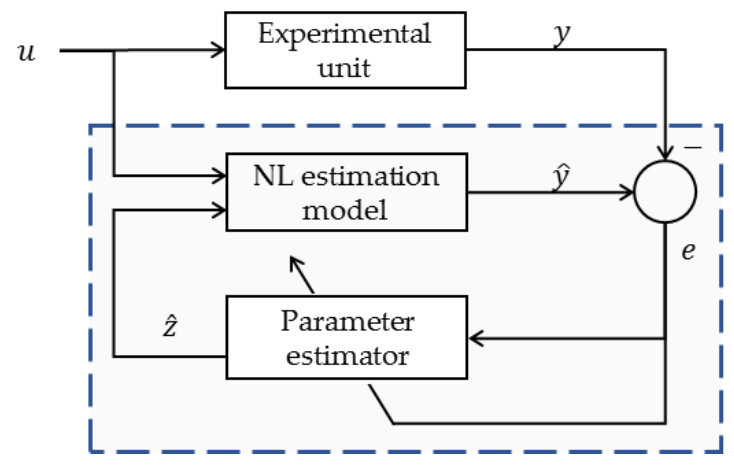

Figure 2. Data-driven parameter estimation architecture.

\section{Results}

$F_{\mathrm{M}}$ is the only measurement obtained continuously. Thus, the global sensitivity analysis and the data-driven estimation are performed using $F_{M}$ as the input signal. The other available measurements $\left(M_{\mathrm{W}}, M_{\mathrm{n}}\right)$ are employed as a reference for comparing the accuracy of the estimated properties.

The system was simulated in MATLAB R2015a, running on a PC Intel Core ${ }^{\mathrm{TM}}$ i7-4790K CPU @ $4.00 \mathrm{GHz}$ with $16.0 \mathrm{~Gb}$ of installed RAM. The ODEs of the system were solved using ode23s [43], based on a modified Rosenbrock formula of order 2 . The sampling time of $F_{\mathrm{M}}$ was every $1 \mathrm{~s}$, and the RCMR algorithm together with the nonlinear model run in approximately $0.0035 \mathrm{~s}$, which makes feasible the online deployment of the proposed strategy. Computational methods can be used for online applications if they are faster than the real process by a factor of 100 [44].

\subsection{Global Sensitivity Analysis}

From the global sensitivity analysis of the seven kinetic parameters $\left(k_{1-7}\right)$, the fifth and seventh show the highest overall sensitivity, as portrayed in Figure 3. This result is consistent because $k_{7}$ is the exponent in the pre-exponential propagation rate constant $\left(k_{0 p}=10^{k_{7}}\right)$ of the Arrhenius equation. The propagation rate constant determines the monomer consumption rate; thus, it strongly influences the value of $F_{\mathrm{M}}$ (ethylene flow rate to the reactor), seeking to maintain the ethylene concentration in the reactor constant during the polymerization. The rate constant for the living chain (catalyst) deactivation $\left(k_{d P}\right)$ is a function of $k_{5}$ (as defined in Table 1 ). Since $k_{d P}$ influences the moment equations, it is expected that this parameter is influential as well. These results are in agreement with the work of Brandão et al. (2017) [13], because $k_{5}$ and $k_{7}$ belong to the parameter set classified as significant when an identifiability analysis was applied over the seven parameters. Figure 3 a illustrates the first-order, and Figure $3 \mathrm{~b}$ the total sensitivity indices when using 100 samples. 


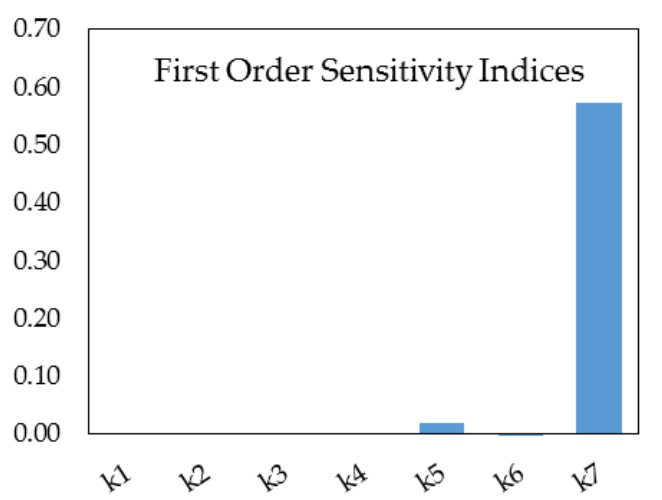

(a)

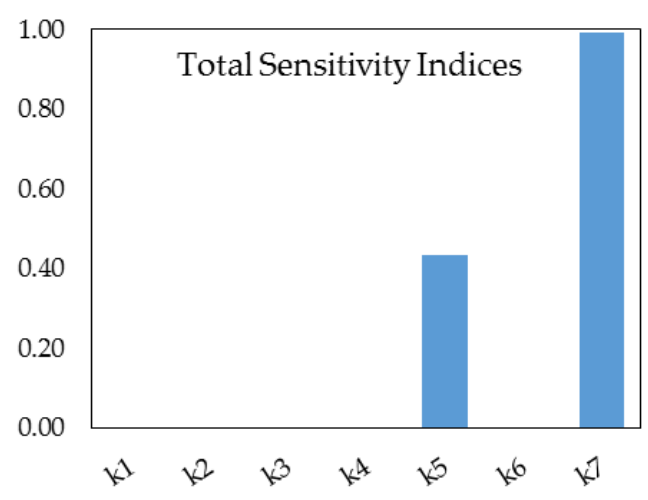

(b)

Figure 3. Global sensitivity indices: (a) first-order sensitivity index; (b) total sensitivity index.

\subsection{Homopolymerization}

Homopolymerization experiments at different reaction temperatures were used to test the RCMR algorithm on its kinetic parameter estimation capabilities. The RCMR algorithm was implemented considering: $\mathbf{P}_{0}=\mathbf{R}_{\boldsymbol{\theta}}{ }^{-1}, \boldsymbol{\theta}_{0}=0$, and $n_{c}=0$.

Initially, only $k_{7}$, and consequently $k_{\mathrm{p}_{11}}$, were estimated, using the initial guess $k_{7}=0$. To improve the convergence of the algorithm it was assumed that $l_{z}=2$, meaning that two parameters were estimated rather than one. For the non-estimated parameter, a constant value of $\hat{z}=11.3$ provided a satisfactory response and tradeoff. With these considerations, the architecture selected was: $n_{f}=2$ so that $l_{\theta}=2, \lambda=0.999$, and

$$
G_{f}(q)=\frac{\left[\begin{array}{ll}
1 & 0
\end{array}\right]}{q}+\frac{\left[\begin{array}{ll}
0 & 1
\end{array}\right]}{q^{2}}
$$

For estimating a single parameter, the algorithm considered $\mathbf{R}_{\theta}=0.01 \mathbf{I}_{l_{\theta}}$, and $\mathbf{R}_{\mathrm{e}}=0.1$.

Figure 4 shows the estimates for $k_{7}$ and $k_{\mathrm{p}_{11}}$ at different polymerization temperatures. The estimated parameters converge, in all cases, close to theoretical values without requiring prior knowledge of the initial value or range of the parameter.

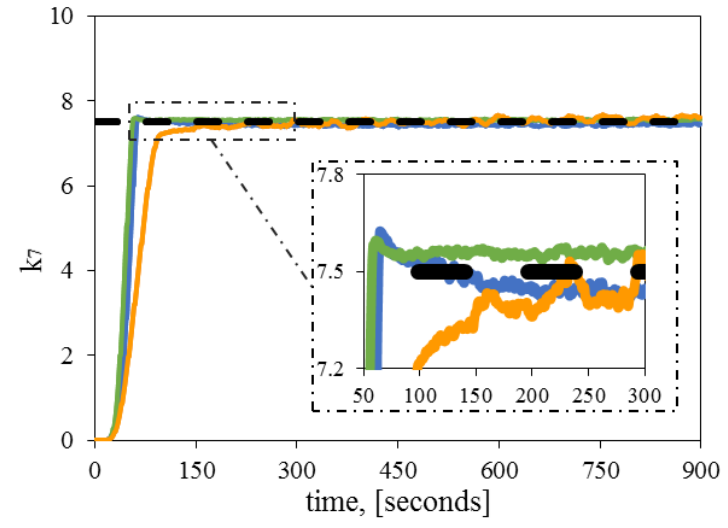

(a)

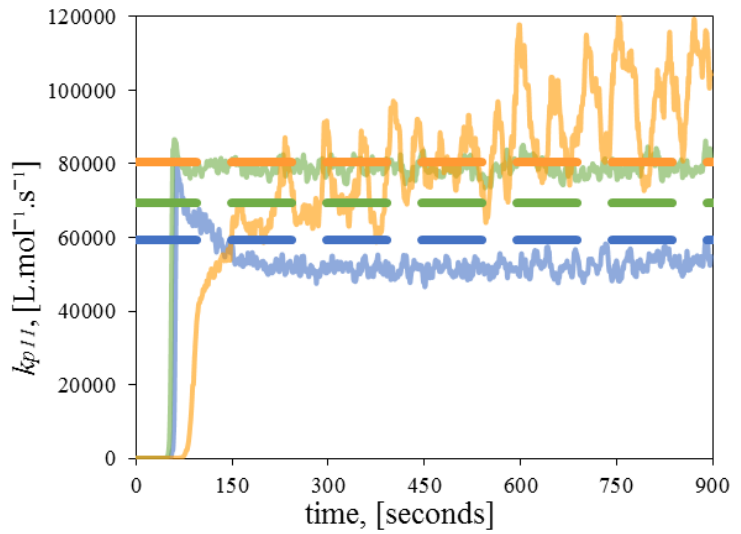

(b)

Figure 4. Estimation of a single significant parameter. Comparison between theoretical (dashed line), and estimated kinetic parameters (continuous line) at $120^{\circ} \mathrm{C}$ (blue), $130{ }^{\circ} \mathrm{C}$ (green) $140{ }^{\circ} \mathrm{C}$ (orange):

(a) Dynamic estimation of $k_{7}$; (b) Dynamic estimation of $k_{\mathrm{p} 11}$.

Furthermore, the most significant kinetic parameters of the system, $k_{5}$ and $k_{7}$, and consequently $k_{d P}$ and $k_{\mathrm{p}_{11}}$, were estimated simultaneously. The same architecture implemented for single parameter estimation was used in the simultaneous estimation, with the distinction that the parameters were 
estimated in separate channels using the same error signal. The non-estimated parameters were different: $\hat{z}_{k_{5}}=23.5$, and $\hat{z}_{k_{7}}=15.0$, and everything else remained the same.

Figure 5 shows the results of the estimated $k_{5}, k_{d P}, k_{7}$, and $k_{\mathrm{p}_{11}}$ at different polymerization temperatures. In all cases, the estimated parameters $\left(k_{5}, k_{7}\right)$ approached their theoretical values, converging from an initial value of 0 in both cases. A noisy response was observed, as in Figure 4, which can be attributed to the presence of impurities that could not be removed during the experiments, or to the occurrence of side reactions not included in the fundamental model. In addition, as the polymerization temperature increased, the estimated parameters became more sensitive to noise, which provided the insight that temperature is proportional to the noise/uncertainty of the experimental data.

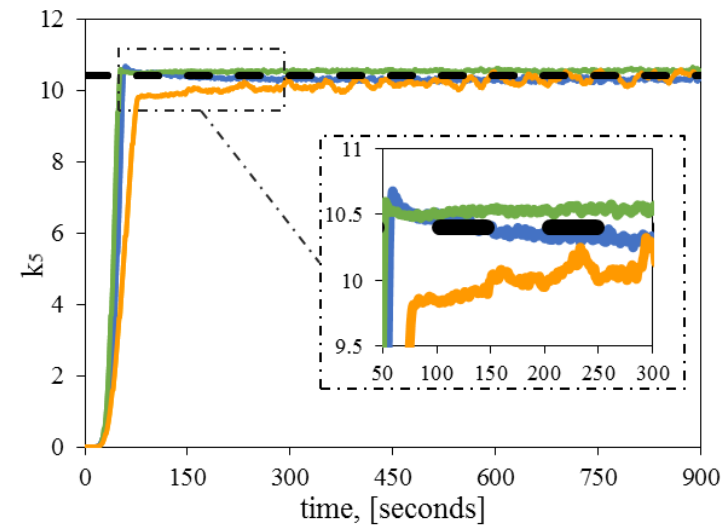

(a)

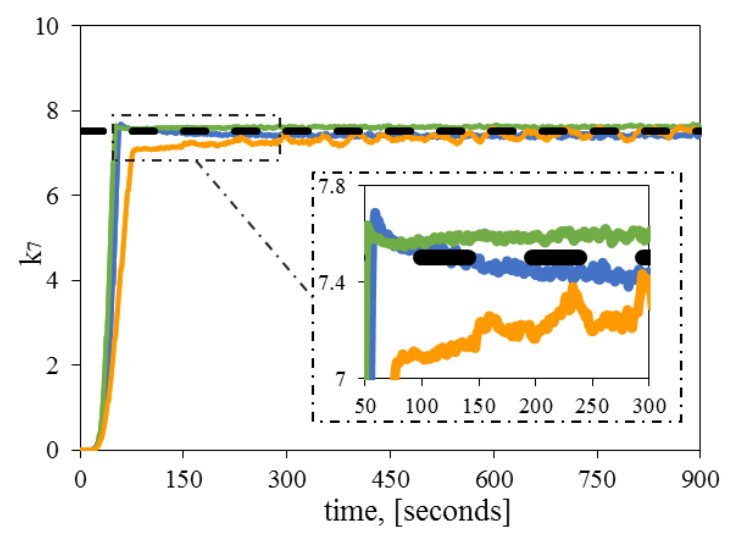

(c)

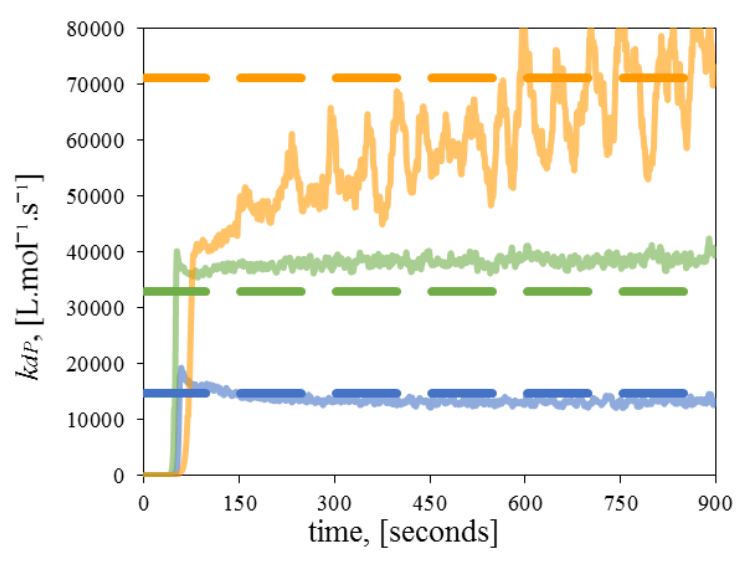

(b)

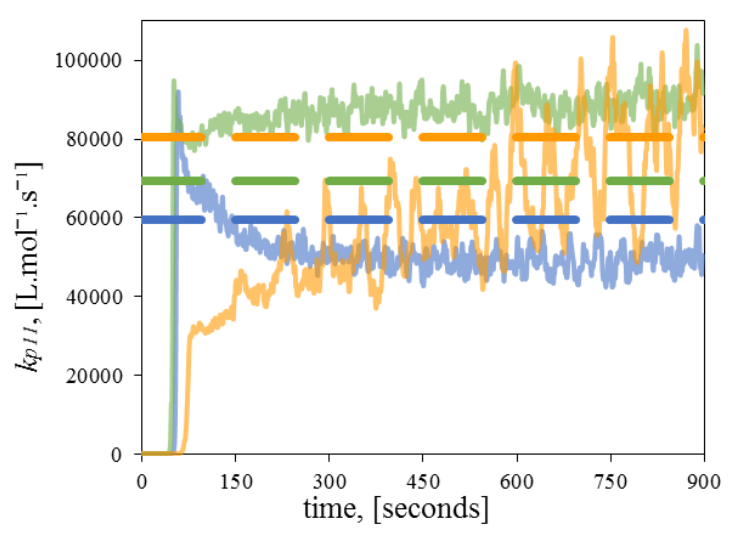

(d)

Figure 5. Estimation of two significant parameters. Comparison between theoretical (dashed line), and estimated kinetic parameters (continuous line) at $120^{\circ} \mathrm{C}$ (blue), $130{ }^{\circ} \mathrm{C}$ (green) $140{ }^{\circ} \mathrm{C}$ (orange): (a) Dynamic estimation of $k_{5}$; (b) Dynamic estimation of $k_{d p}$; (c) Dynamic estimation of $k_{7}$, (d) Dynamic estimation of $k_{\mathrm{p} 11}$.

Figure 6 illustrates how the RCMR can both estimate $F_{M}$ and reduce the measurement's noise. The reader should note that it takes some time for the estimated $F_{M}$ to achieve its expected value. Goel and Bernstein (2018) [13] explained that the unknown parameter moves towards different subspaces until it tends to the subspace spanned by $N_{1}{ }^{T}$. In addition, there is a delay time difference between the estimated $\hat{F}_{\mathrm{M}}$ with a single and two parameters, which is mostly related to the tuning. Finally, as stated before, the $\hat{F}_{\mathrm{M}}$ at the highest temperature $\left(140{ }^{\circ} \mathrm{C}\right)$ shows an oscillatory response. 


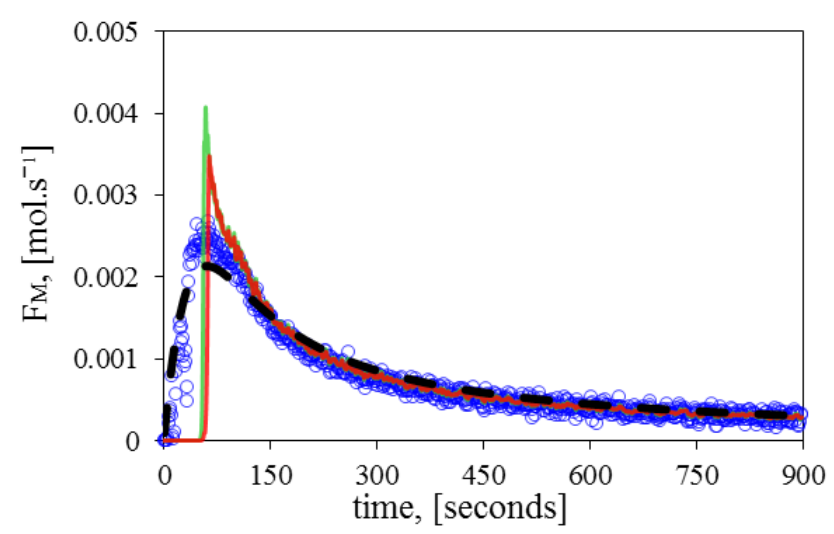

(a)

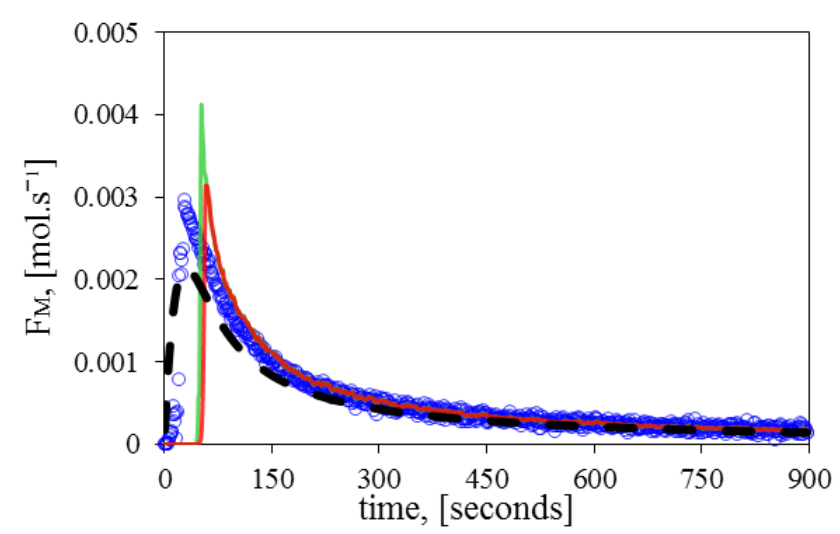

(b)

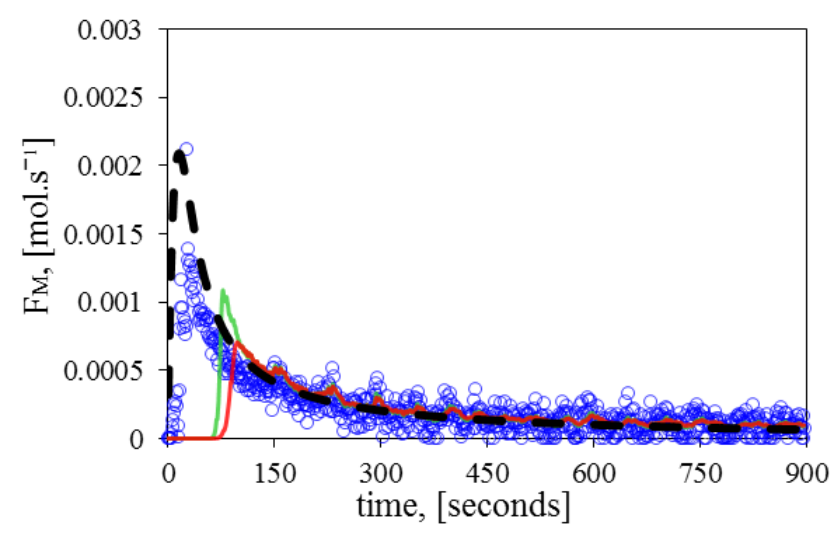

(c)

Figure 6. Comparison of the monomer flow rate $\left(F_{\mathrm{M}}\right)$ between the experimental values (circles), fundamental model (dashed line), estimated with a single parameter (continuous red line), and estimated with two parameters (continuous green line) at: (a) $120^{\circ} \mathrm{C}$, (b) $130{ }^{\circ} \mathrm{C}$, (c) $140{ }^{\circ} \mathrm{C}$.

Figure 7 compares the results computed by the nonlinear model, the measured results, and the estimated average properties at different polymerization temperatures. The results at $120^{\circ} \mathrm{C}$ of both $M_{\mathrm{w}}$ and $M_{\mathrm{n}}$ are very close to their theoretical and experimental values, but as the temperature increases, the uncertainty also increases. The estimation of two parameters simultaneously appears to provoke less reliable results when compared to the estimated properties with a single parameter, especially at higher temperatures. 


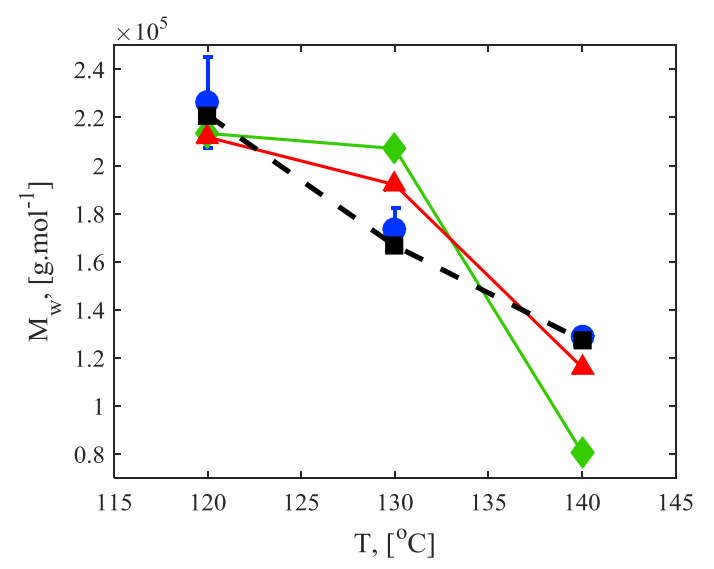

(a)

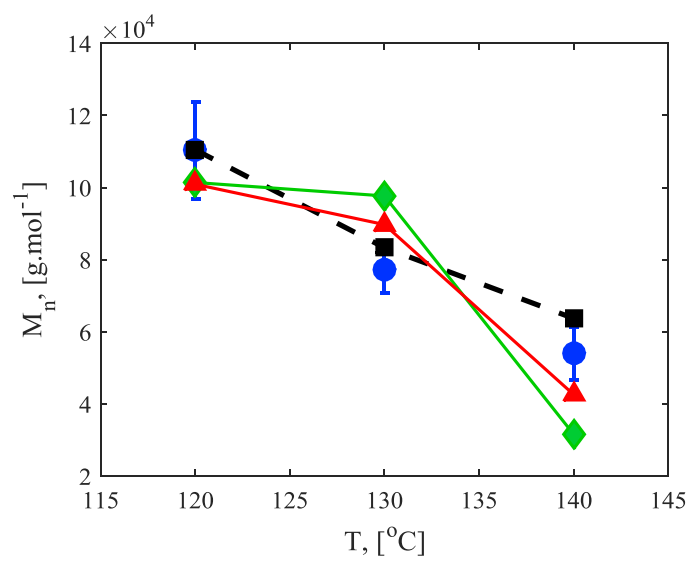

(b)

Figure 7. Comparison of the final average properties at different temperatures for experimental values (circles), fundamental model (squares-dashed line), estimated with a single parameter (triangles-continuous line), and estimated with two parameters (diamonds-continuous line): (a) weight-average molecular weight $\left(M_{\mathrm{W}}\right)$; (b) number-average molecular weight $\left(M_{\mathrm{n}}\right)$.

\subsection{Copolymerization}

Following the criterion used for the homopolymerization experiments, the copolymerizations used the same RCMR architecture for the estimation of significant kinetic parameters and important polymer properties. Indeed, the criterion extends to the application of the same channels for estimating one and two significant kinetic parameters and their resulting properties.

The copolymerization experiments considered only one temperature. Initially, $k_{7}$ and consequently $k_{\mathrm{p}_{11}}$ were estimated for the copolymerizations described in Table 4a,b. Figure 8 illustrates the results of the estimated $k_{7}$ and $k_{\mathrm{p}_{11}}$ at $120{ }^{\circ} \mathrm{C}$ and different initial diene concentrations. The unknown parameter converges towards the theoretical value without requiring prior knowledge at both initial diene concentrations.

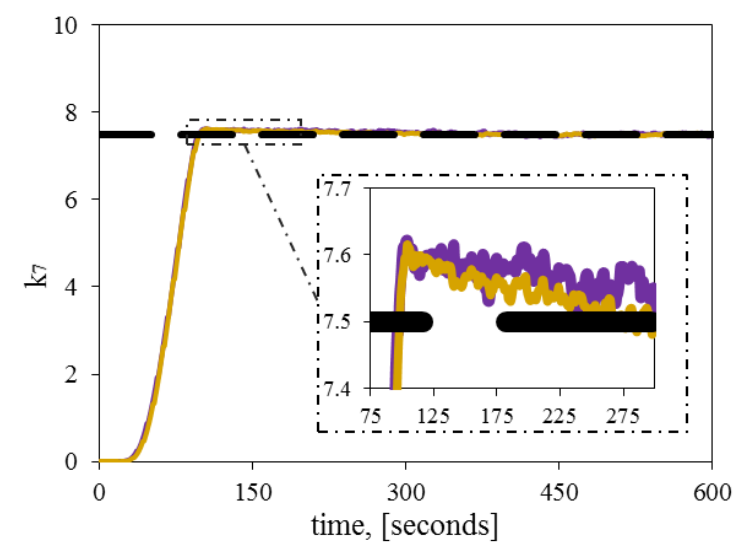

(a)

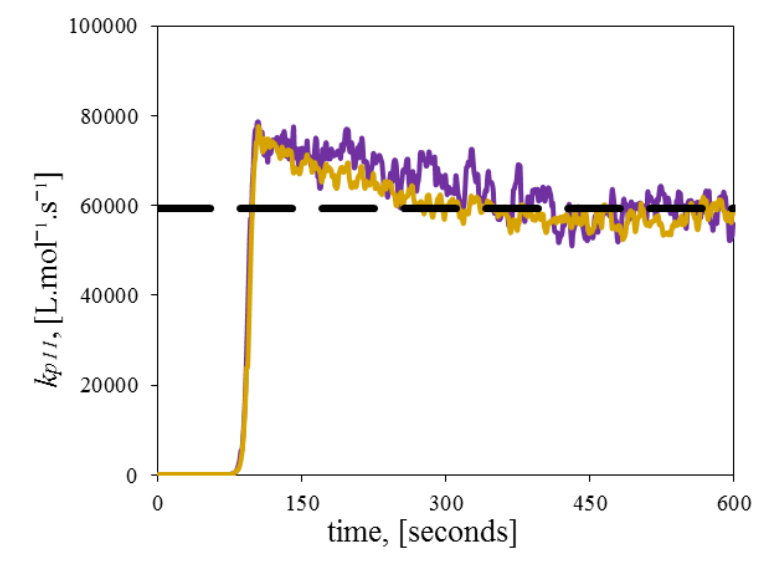

(b)

Figure 8. Estimation of a single significant parameter. Comparison between theoretical (dashed line), and estimated kinetic parameters (continuous line) at different initial diene concentrations for the copolymerization experiment A (purple), and copolymerization experiment B (gold); (a) Dynamic estimation of $k_{7} ;(\mathbf{b})$ Dynamic estimation of $k_{\mathrm{p} 11}$. 
Moreover, the parameters $k_{5}$ and $k_{7}$, and, consequently, $k_{d P}$ and $k_{\mathrm{p}_{11}}$, were estimated simultaneously, using the same architecture and tuning used in the homopolymerizations. Figure 9 shows the results of the estimated $k_{5}, k_{d p}, k_{7}$, and $k_{\mathrm{p}_{11}}$ at different initial diene concentrations at $120{ }^{\circ} \mathrm{C}$. In all cases, the unknown parameters $k_{5}$ and $k_{7}$ approach the theoretical values, starting from an initial guess of 0 . An interesting observation in these experiments is the slight decreasing trend (negative slope) of the parameters, made clearer in the dynamic evolution of $k_{d P}$ and $k_{\mathrm{p}_{11}}$. The reason for this behavior might be related to LCBs formation during the copolymerization. The presence of LCBs in the living chains might cause a steric hindrance to the incorporation of ethylene molecules, which disfavors deactivation and propagation reactions. However, this hypothesis must be proved by additional experiments and further simulations.

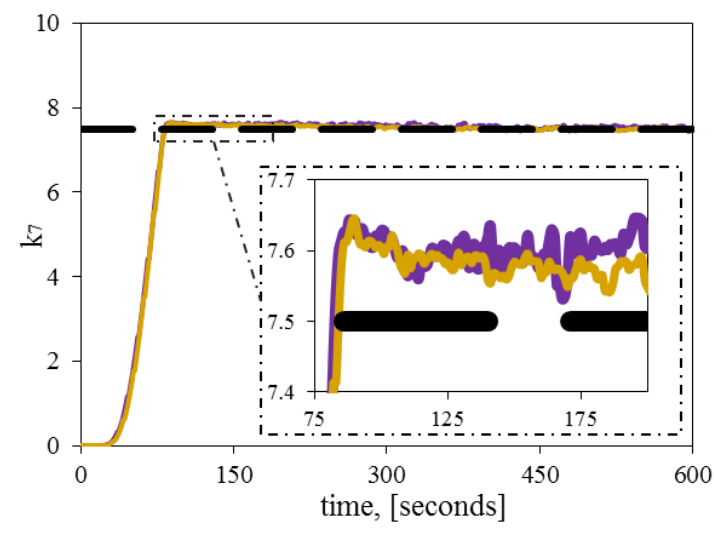

(a)

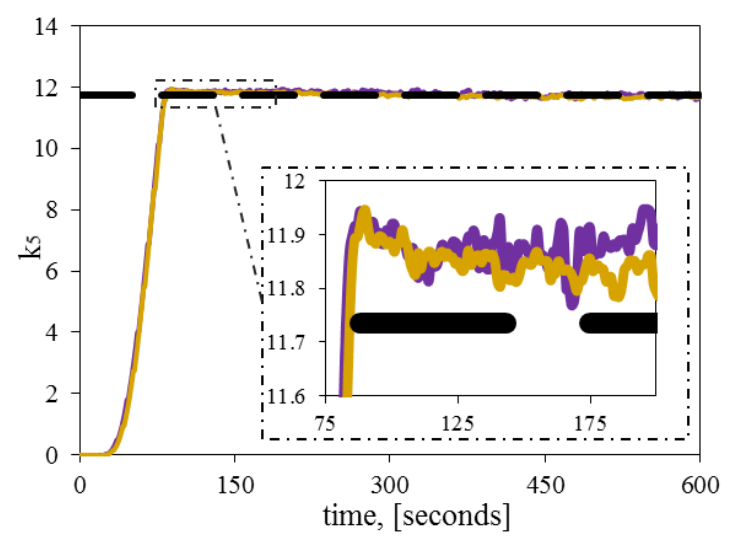

(c)

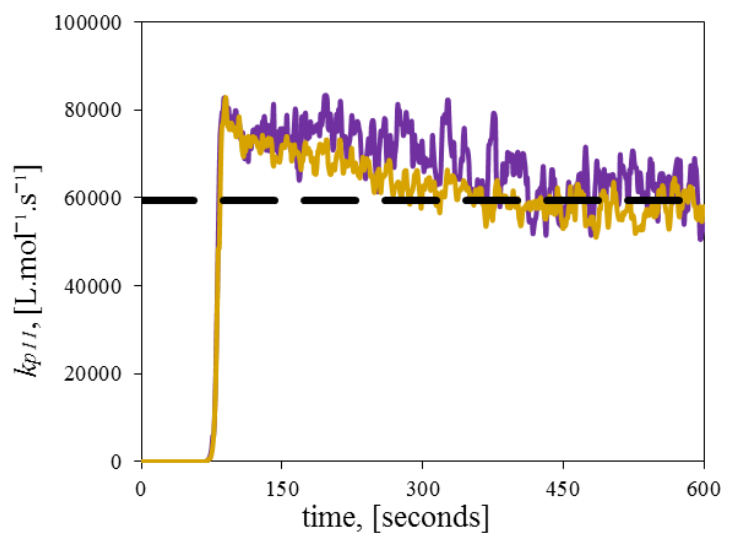

(b)

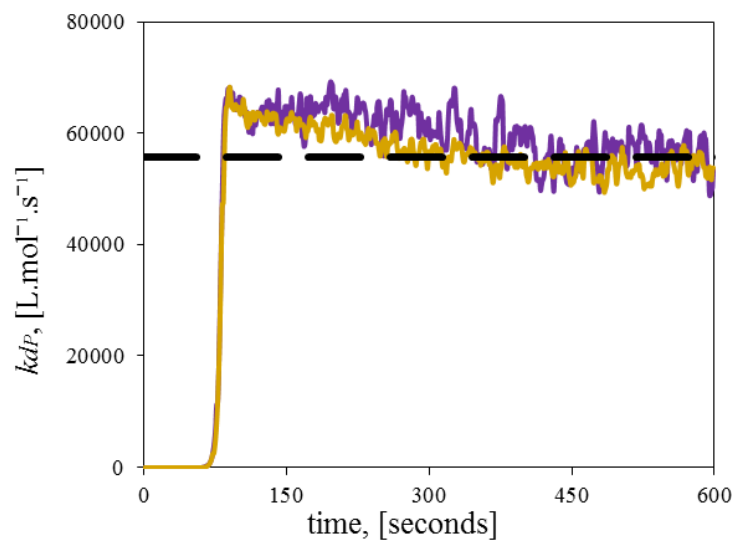

(d)

Figure 9. Estimation of two significant parameters. Comparison between theoretical (dashed line), and estimated kinetic parameters (continuous line) at different initial diene concentrations for the copolymerization experiment A (purple), and copolymerization experiment B (gold): (a) Dynamic estimation of $k_{7} ;(\mathbf{b})$ Dynamic estimation of $k_{\mathrm{p} 11} ;$ (c) Dynamic estimation of $k_{5} ;(\mathbf{d})$ Dynamic estimation of $k_{\mathrm{d} P}$.

Figure 10 demonstrates how the RCMR can estimate $F_{M}$ effectively and reduce the measurement's noise. As before, it takes some time for the estimated $F_{M}$ to achieve values close to experimental and theoretical values. There are no visible differences observed when the initial concentration of diene varies, which makes it possible to conclude that temperature is more influential on the reaction behavior. In contrast to the homopolymerization results (Figure 6), the estimated $F_{M}$ in the copolymerization experiments shows a higher delay time of convergence. It could be argued that it should be influenced by the increase in the complexity of modelling, but because the RCMR algorithm is a purely data-driven 
strategy, which does not require information on the nonlinear model, the reasons must be totally related to the nature of the experiment.

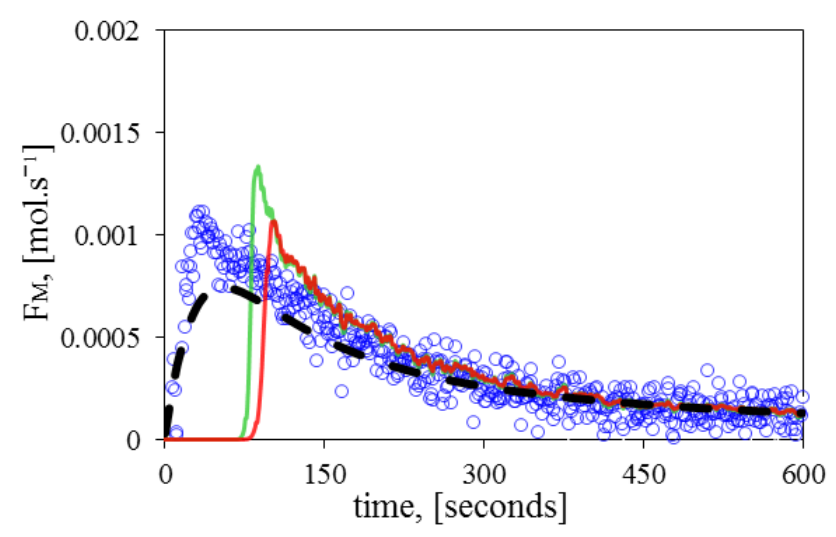

(a)

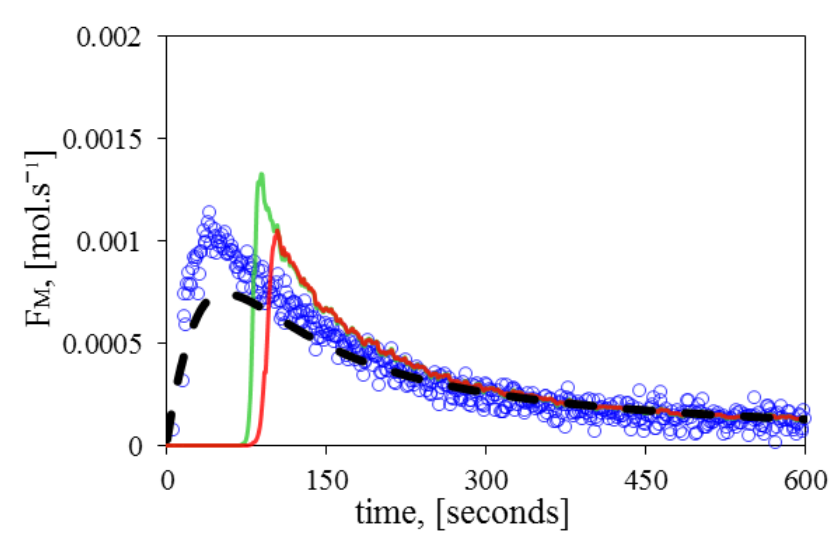

(b)

Figure 10. Comparison of the monomer flow rate $\left(F_{\mathrm{M}}\right)$ during copolymerization between the experimental values (circles), fundamental model (dashed line), estimated with a single parameter (continuous red line), and estimated with two parameters (continuous green line) at different initial diene concentrations: (a) copolymerization experiment $\mathrm{A},(\mathbf{b})$ copolymerization experiment $\mathrm{B}$.

Finally, Figure 11 compares the results computed by the fundamental model, the measured results, and the estimated average properties of the copolymerization experiments at different initial diene concentrations. Data on the average properties was obtained during the polymerization experiments. The results show that the RCMR algorithm, besides computing the unknown parameters, can estimate $M_{\mathrm{n}}$ satisfactorily using the error signal obtained as the difference between the estimated and measured $F_{\mathrm{M}}$. This signal allows the estimator to gain enough information on the system to estimate $M_{n}$. Of course, the estimation additionally relies on the correctness of the model and the non-estimated parameters as well, but the obtained results are comparable with theoretical and experimental values. On the other hand, $M_{\mathrm{w}}$ achieves a similar dynamic when compared to the fundamental model, but the estimates fail to attain perfect values close to the experimental measurements. 


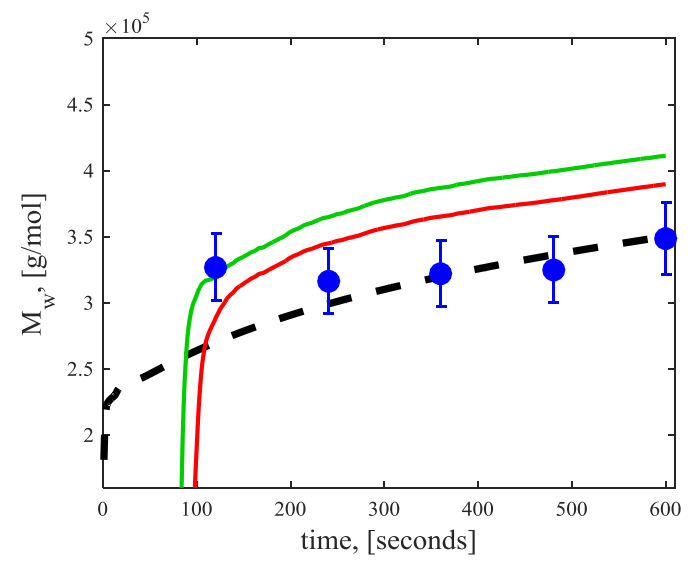

(a)

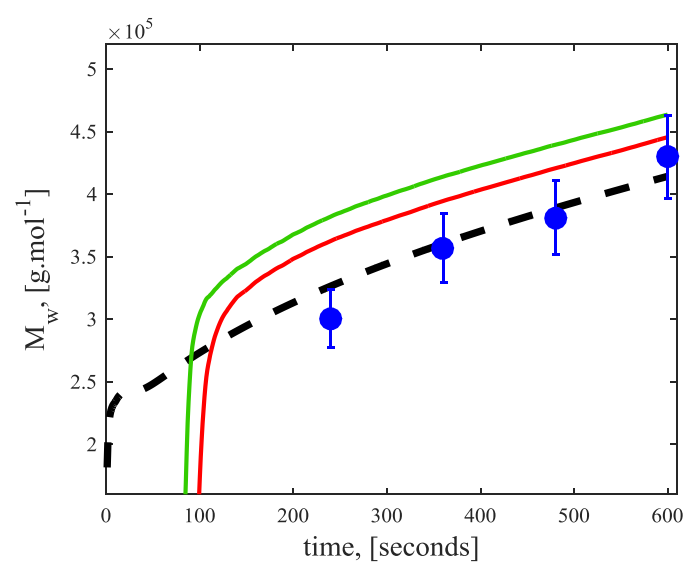

(c)

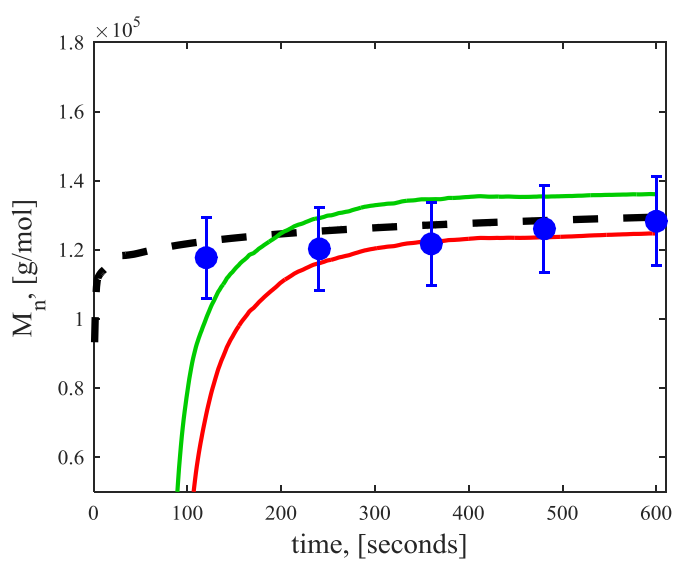

(b)

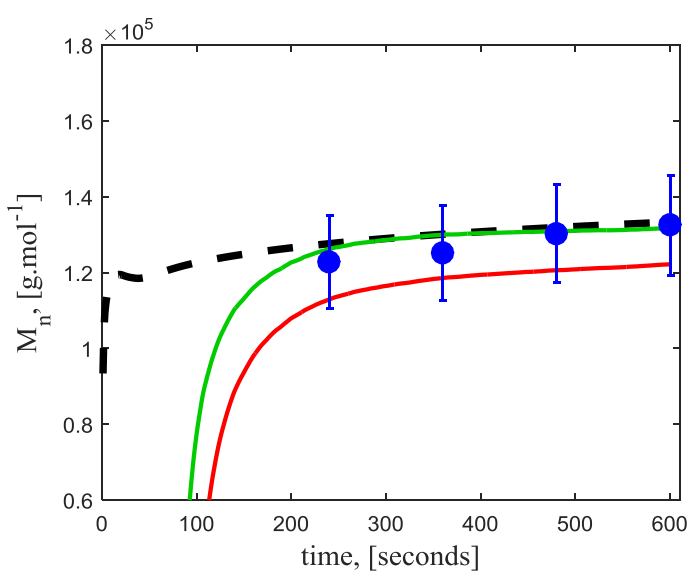

(d)

Figure 11. Comparison of average polymer properties at different initial diene concentrations between experimental values (circles), fundamental model (dashed line), estimated with a single parameter (red continuous line), and estimated with two parameters (green continuous line).

(a) Weight-average molecular weight $\left(M_{\mathrm{W}}\right)$ for copolymerization A; (b) number-average molecular weight $\left(M_{\mathrm{n}}\right)$ for copolymerization $\mathrm{A}$; (c) weight-average molecular weight $\left(M_{\mathrm{w}}\right)$ for copolymerization

B; (d) number-average molecular weight $\left(M_{n}\right)$ for copolymerization B.

\section{Conclusions}

In this contribution, a data-driven strategy for the online estimation of important kinetic parameters was assessed and implemented for the copolymerization of ethylene with 1,9-decadiene using dimethylsilyl (N-tert-butylamido) (tetramethylcyclopentadienyl) titanium dichloride (CGC)/MAO as catalyst. A global sensitivity analysis was performed initially to all polymerization kinetic parameters. The first and total sensitivity indices made it possible to choose the significant parameters of the model. Thereafter, the RCMR algorithm, a strategy never implemented in polymerization applications, permitted the estimation of the significant kinetic parameters, which were assumed to be unknown. After verifying consistency, the proposed strategy was tested in the copolymerization of ethylene with 1,9-decadiene at different diene concentrations. Overall, results were satisfactory, showing not only adequacy in signal processing, but also in parameter and property estimation.

The usage of data-driven algorithms such as the RCMR represents a paradigm that could permit easier estimation of parameters of nonlinear systems, such as those observed in polymer synthesis. Disturbances in the experimental data (e.g., impurities, experimental errors, and less frequent side reactions) that might not be captured by the fundamental model could be overcome by applying this strategy. 
The proposed strategy also holds the advantage that it is capable of being adapted to the conditions of the experiment, and it can estimate the important kinetic parameters of the system while the reaction is running. This could be beneficial for processes where the major kinetic parameters are unknown for all the different types of catalysts, or the composition of the catalysts might be slightly different from each other, resulting in important differences. In the particular case of the RCMR algorithm, it has the additional advantage of estimating the parameters from an initial value of zero without requiring knowledge of the model.

Future work in this field will include the evaluation of the proposed framework and estimation algorithm in other chemical processes and polymerization systems.

Author Contributions: S.D.S. designed the framework that combines process modelling, global sensitivity analysis, and the RCMR algorithm under the supervision of J.A.R.; A.L.T.B. collected the experimental data and reviewed the reaction mechanism and the process modelling in collaboration with J.B.P.S.; S.D.S. wrote the article, while everyone contributed in reviewing and enriching the content.

Funding: This research received no external funding.

Acknowledgments: The authors acknowledge Ankit Goel from the University of Michigan for providing guidelines that facilitated the implementation of the RCMR algorithm.

Conflicts of Interest: The authors declare no conflict of interest.

\section{Nomenclature}

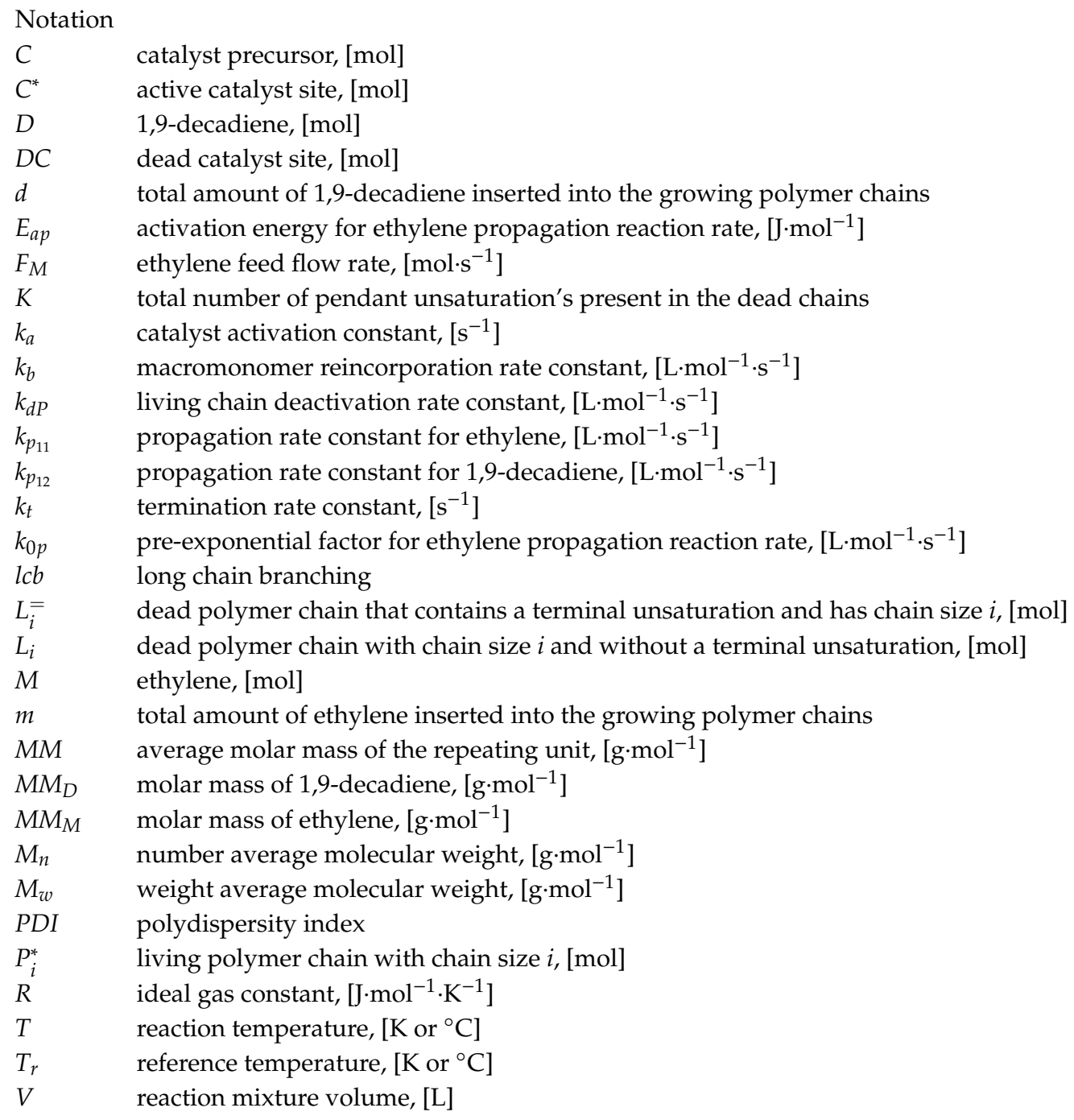


Greek letters

$\lambda_{k} \quad k^{\text {th }}$ moment for the dead chain

$\mu_{k} \quad k^{\text {th }}$ moment for the living chain

$\rho_{P E} \quad$ Polyethylene density, $\left[\mathrm{g} \cdot \mathrm{L}^{-1}\right]$

$\varphi \quad$ Average frequency of pendant double bonds in the polymer chains

\section{References}

1. Liu, P.; Liu, W.; Wang, W.J.; Li, B.G.; Zhu, S. A Comprehensive Review on Controlled Synthesis of Long-Chain Branched Polyolefins: Part 1, Single Catalyst Systems. Macromol. React. Eng. 2016, 10, 156-179. [CrossRef]

2. Stadler, F.J.; Piel, C.; Klimke, K.; Kaschta, J.; Parkinson, M.; Wilhelm, M.; Münstedt, H. Influence of type and content of various comonomers on long-chain branching of ethene/ $\alpha$-olefin copolymers. Macromolecules 2006, 39, 1474-1482. [CrossRef]

3. Soares, J.B.; Hamielec, A.E. Bivariate chain length and long chain branching distribution for copolymerization of olefins and polyolefin chains containing terminal double-bonds. Macromol. Theory Simul. 1996, 5, 547-572. [CrossRef]

4. Wang, W.J.; Yan, D.; Zhu, S.; Hamielec, A.E. Kinetics of long chain branching in continuous solution polymerization of ethylene using constrained geometry metallocene. Macromolecules 1998, 31, 8677-8683. [CrossRef]

5. Chum, P.S.; Kruper, W.J.; Guest, M.J. Materials properties derived from INSITE metallocene catalysts. Adv. Mater. 2000, 12, 1759-1767. [CrossRef]

6. Choo, T.N.; Waymouth, R.M. Cyclocopolymerization: A mechanistic probe for dual-site alternating copolymerization of ethylene and $\alpha$-olefins. J. Am. Chem. Soc. 2002, 124, 4188-4189. [CrossRef]

7. Naga, N.; Toyota, A. Unique Insertion Mode of 1,7-Octadiene in Copolymerization with Ethylene by a Constrained-Geometry Catalyst. Macromol. Rapid Commun. 2004, 25, 1623-1627. [CrossRef]

8. Mehdiabadi, S.; Soares, J.B. Production of Ethylene/ $\alpha$-Olefin/1, 9-Decadiene Copolymers with Complex Microstructures Using a Two-Stage Polymerization Process. Macromolecules 2011, 44, 7926-7939. [CrossRef]

9. Soares, J.B. Mathematical Modeling of the Long-Chain Branch Structure of Polyolefins Made with Two Metallocene Catalysts: An Algebraic Solution. Macromol. Theory Simul. 2002, 11, 184-198. [CrossRef]

10. Ferreira, L.C., Jr.; Nele, M.; Costa, M.A.; Pinto, J.C. Mathematical Modeling of MWD and CBD in Polymerizations with Macromonomer Reincorporation and Chain Running. Macromol. Theory Simul. 2010, 19, 496-513. [CrossRef]

11. Mogilicharla, A.; Mitra, K.; Majumdar, S. Modeling of propylene polymerization with long chain branching. Chem. Eng. J. 2014, 246, 175-183. [CrossRef]

12. Albeladi, A.; Mehdiabadi, S.; Soares, J.B. Modeling Possible Long Chain Branching Reactions for Polyethylene in a Semi-Batch Reactor. 2018. Available online: http://dc.engconfintl.org/prex/49 (accessed on 26 February 2019).

13. Brandão, A.L.; Alberton, A.L.; Pinto, J.C.; Soares, J.B. Copolymerization of Ethylene with 1, 9-Decadiene: Part I-Prediction of Average Molecular Weights and Long-Chain Branching Frequencies. Macromol. Theory Simul. 2017, 26, 1600059. [CrossRef]

14. Brandão, A.L.; Alberton, A.L.; Pinto, J.C.; Soares, J.B. Copolymerization of Ethylene with 1, 9-Decadiene: Part II-Prediction of Molecular Weight Distributions. Macromol. Theory Simul. 2017, 26, 1700040. [CrossRef]

15. Soares, J.B.; McKenna, T.F. Polyolefin Reaction Engineering; John Wiley \& Sons: Hoboken, NJ, USA, 2013.

16. Qin, S.J. Process data analytics in the era of big data. AIChE J. 2014, 60, 3092-3100. [CrossRef]

17. Salas, S.D.; Ghadipasha, N.; Zhu, W.; Mcafee, T.; Zekoski, T.; Reed, W.F.; Romagnoli, J.A. Framework Design for Weight-Average Molecular Weight Control in Semi-Batch Polymerization. Control Eng. Pract. 2018, 78, 12-23. [CrossRef]

18. Kozub, D.J.; MacGregor, J.F. State estimation for semi-batch polymerization reactors. Chem. Eng. Sci. 1992, 47, 1047-1062. [CrossRef]

19. Tatiraju, S.; Soroush, M. Nonlinear state estimation in a polymerization reactor. Ind. Eng. Chem. Res. 1997, 36, 2679-2690. [CrossRef]

20. Lopez, T.; Alvarez, J. On the effect of the estimation structure in the functioning of a nonlinear copolymer reactor estimator. J. Process Control 2004, 14, 99-109. [CrossRef] 
21. Galdeano, R.; Asteasuain, M.; Sánchez, M.C. Unscented transformation-based filters: Performance comparison analysis for the state estimation in polymerization processes with delayed measurements. Macromol. React. Eng. 2011, 5, 278-293. [CrossRef]

22. Hashemi, R.; Kohlmann, D.; Engell, S. Optimizing control and state estimation of a continuous polymerization process in a tubular reactor with multiple side-streams. Macromol. React. Eng. 2016, 10, 415-434. [CrossRef]

23. Zoellner, K.; Reichert, K.H. Gas phase polymerization of butadiene-Kinetics, particle size distribution, modeling. Chem. Eng. Sci. 2001, 56, 4099-4106. [CrossRef]

24. Ghadipasha, N.; Geraili, A.; Romagnoli, J.A.; Castor, C.A.; Drenski, M.F.; Reed, W.F. Combining on-line characterization tools with modern software environments for optimal operation of polymerization processes. Processes 2016, 4, 5. [CrossRef]

25. Schwaab, M.; Biscaia, E.C., Jr.; Monteiro, J.L.; Pinto, J.C. Nonlinear parameter estimation through particle swarm optimization. Chem. Eng. Sci. 2008, 63, 1542-1552. [CrossRef]

26. Salas, S.D.; Romagnoli, J.A.; Tronci, S.; Baratti, R. A geometric observer design for a semi-batch free-radical polymerization system. Comput. Chem. Eng. 2019, 126, 391-402. [CrossRef]

27. Sirohi, A.; Choi, K.Y. On-line parameter estimation in a continuous polymerization process. Ind. Eng. Chem. Res. 1996, 35, 1332-1343. [CrossRef]

28. Li, R.; Corripio, A.B.; Henson, M.A.; Kurtz, M.J. On-line state and parameter estimation of EPDM polymerization reactors using a hierarchical extended Kalman filter. J. Process Control 2004, 14, 837-852. [CrossRef]

29. Chen, T.; Morris, J.; Martin, E. Particle filters for state and parameter estimation in batch processes. J. Process Control 2005, 15, 665-673. [CrossRef]

30. Sheibat-Othman, N.; Peycelon, D.; Othman, S.; Suau, J.M.; Fevotte, G. Nonlinear observers for parameter estimation in a solution polymerization process using infrared spectroscopy. Chem. Eng. J. 2008, 140, 529-538. [CrossRef]

31. Salas Ortiz, S.D. A Model-Based Framework for the Smart Manufacturing of Polymers. Ph.D. Thesis, Louisiana State University and Agricultural and Mechanical College, Baton Rouge, LA, USA, 2019; p. 4897.

32. Wu, Q.L.; Cournede, P.H.; Mathieu, A. An efficient computational method for global sensitivity analysis and its application to tree growth modelling. Reliab. Eng. Syst. Saf. 2012, 107, 35-43. [CrossRef]

33. Goel, A.; Duraisamy, K.; Bernstein, D.S. Parameter estimation in the burgers equation using retrospective-cost model refinement. In Proceedings of the American Control Conference (ACC), Boston, MA, USA, 6-8 July 2016; pp. 6983-6988.

34. Goel, A.; Bernstein, D.S. Parameter estimation for nonlinearly parameterized gray-box models. In Proceedings of the Annual American Control Conference (ACC), Milwaukee, WI, USA, 27-29 June 2018; pp. 5280-5285.

35. Goel, A.; Bernstein, D.S. Data-Driven Parameter Estimation for Models with Nonlinear Parameter Dependence. In Proceedings of the 2018 IEEE Conference on Decision and Control (CDC), Miami Beach, FL, USA, 17-19 December 2018; pp. 1470-1475.

36. Hulburt, H.M.; Katz, S. Some problems in particle technology: A statistical mechanical formulation. Chem. Eng. Sci. 1964, 19, 555-574. [CrossRef]

37. Eberhart, R.; Kennedy, J. Particle Swarm Optimization. In Proceedings of the IEEE International Conference on Neural Networks, Perth, Australia, 27 November-1 December 1995; pp. 1942-1948.

38. Sobol, I.M. Sensitivity estimates for nonlinear mathematical models. Math. Model. Comput. Exp. 1993, 1, 407-414.

39. Cosenza, A.; Mannina, G.; Vanrolleghem, P.A.; Neumann, M.B. Variance-based sensitivity analysis for wastewater treatment plant modelling. Sci. Total Environ. 2014, 470, 1068-1077. [CrossRef]

40. Saltelli, A. Making best use of model evaluations to compute sensitivity indices. Comput. Phys. Commun. 2002, 145, 280-297. [CrossRef]

41. Saltelli, A.; Ratto, M.; Andres, T.; Campolongo, F.; Cariboni, J.; Gatelli, D.; Tarantola, S. Global Sensitivity Analysis: The Primer; John Wiley \& Sons: Hoboken, NJ, USA, 2008.

42. Salas, S.D.; Geraili, A.; Romagnoli, J.A. Optimization of Renewable Energy Businesses under Operational Level Uncertainties through Extensive Sensitivity Analysis and Stochastic Global Optimization. Ind. Eng. Chem. Res. 2017, 56, 3360-3372. [CrossRef] 
43. Shampine, L.F.; Reichelt, M.W. The matlab ode suite. SIAM J. Sci. Comput. 1997, 18, 1-22. [CrossRef]

44. Porru, M.; Ożkan, L. Monitoring of Batch Industrial Crystallization with Growth, Nucleation, and Agglomeration. Part 2: Structure Design for State Estimation with Secondary Measurements. Ind. Eng. Chem. Res. 2017, 56, 9578-9592. [CrossRef] 